\title{
Distributed Algorithms for Computing Very Large Thresholded Covariance Matrices
}

\author{
ZEKAI J. GAO and CHRIS JERMAINE, Rice University
}

\begin{abstract}
Computation of covariance matrices from observed data is an important problem, as such matrices are used in applications such as principal component analysis (PCA), linear discriminant analysis (LDA), and increasingly in the learning and application of probabilistic graphical models. However, computing an empirical covariance matrix is not always an easy problem. There are two key difficulties associated with computing such a matrix from a very high-dimensional dataset. The first problem is over-fitting. For a $p$-dimensional covariance matrix, there are $p(p-1) / 2$ unique, off-diagonal entries in the empirical covariance matrix $\hat{\mathbf{S}}$; for large $p$ (say, $p>10^{5}$ ), the size $n$ of the dataset is often much smaller than the number of covariances to compute. Over-fitting is a concern in any situation in which the number of parameters learned can greatly exceed the size of the dataset. Thus, there are strong theoretical reasons to expect that for high-dimensional data-even Gaussian data - the empirical covariance matrix is not a good estimate for the true covariance matrix underlying the generative process. The second problem is computational. Computing a covariance matrix takes $O\left(n p^{2}\right)$ time. For large $p$ (greater than 10,000) and $n$ much greater than $p$, this is debilitating. In this article, we consider how both of these difficulties can be handled simultaneously. Specifically, a key regularization technique for high-dimensional covariance estimation is thresholding, in which the smallest or least significant entries in the covariance matrix are simply dropped and replaced with the value 0 . This suggests an obvious way to address the computational difficulty as well: First, compute the identities of the $K$ entries in the covariance matrix that are actually important in the sense that they will not be removed during thresholding, and then in a second step, compute the values of those entries. This can be done in $O(K n)$ time. If $K \ll p^{2}$ and the identities of the important entries can be computed in reasonable time, then this is a big win. The key technical contribution of this article is the design and implementation of two different distributed algorithms for approximating the identities of the important entries quickly, using sampling. We have implemented these methods and tested them using an 800-core compute cluster. Experiments have been run using real datasets having millions of data points and up to 40,000 dimensions. These experiments show that the proposed methods are both accurate and efficient.
\end{abstract}

Categories and Subject Descriptors: H.2.8 [Database Management]: Database Applications

General Terms: Algorithms, Design, Performance

Additional Key Words and Phrases: Covariance matrices, thresholding, distributed algorithm, sampling, text processing

ACM Reference Format:

Zekai J. Gao and Chris Jermaine. 2016. Distributed algorithms for computing very large thresholded covariance matrices. ACM Trans. Knowl. Discov. Data 11, 2, Article 12 (November 2016), 25 pages.

DOI: http://dx.doi.org/10.1145/2935750

This article was supported by the National Science Foundation under grant number 1409543.

Authors' addresses: Z. J. Gao (corresponding author) and C. Jermaine, Computer Science Department, Rice University, Houston, TX 77005; emails: \{jacobgao, cmj4\}@rice.edu.

Permission to make digital or hard copies of part or all of this work for personal or classroom use is granted without fee provided that copies are not made or distributed for profit or commercial advantage and that copies show this notice on the first page or initial screen of a display along with the full citation. Copyrights for components of this work owned by others than ACM must be honored. Abstracting with credit is permitted. To copy otherwise, to republish, to post on servers, to redistribute to lists, or to use any component of this work in other works requires prior specific permission and/or a fee. Permissions may be requested from Publications Dept., ACM, Inc., 2 Penn Plaza, Suite 701, New York, NY 10121-0701 USA, fax +1 (212) 869-0481, or permissions@acm.org.

(c) 2016 ACM 1556-4681/2016/11-ART12 $\$ 15.00$

DOI: http://dx.doi.org/10.1145/2935750 


\section{INTRODUCTION}

Computation of covariance matrices from observed data is an important problem. Such matrices are used in applications such as principal component analysis [Jolliffe 2005], linear discriminant analysis [Izenman 2008], and increasingly in the learning and application of probabilistic graphical models [Koller and Friedman 2009] such as Markov random fields [Kindermann et al. 1980].

Why computing a covariance matrix is difficult. Although useful, computing such a matrix is a deceptively simple problem, for two main reasons. First, there is the problem of over-fitting, which in this context means finding spurious correlations in the data that are not there in the underlying covariance matrix. For a $p$-dimensional covariance matrix, there are $p(p-1) / 2$ unique, off-diagonal entries in the empirical covariance matrix $\hat{\mathbf{S}}$; for large $p$ (say, $p>10^{5}$ ), the size $n$ of the dataset is often much smaller than the number of covariances to compute. Over-fitting is a concern in any situation in which the number of parameters learned can greatly exceed the size of the dataset. Thus, there are strong theoretical reasons to expect that for high-dimensional dataeven Gaussian data-the empirical covariance matrix is not a good estimate for the true covariance matrix underlying the generative process [Marčenko and Pastur1967; Johnstone 2001].

The second problem is computational. An entry $\hat{s}_{j, k}$ in the empirical covariance matrix $\hat{\mathbf{S}}$ is computed as follows:

$$
\hat{s}_{j, k}=\frac{1}{n}\left(\left(\sum_{i} x_{i, j} x_{i, k}\right)-\frac{1}{n}\left(\sum_{i} x_{i, j}\right)\left(\sum_{i} x_{i, k}\right)\right) .
$$

Here, $x_{i, j}$ refers to the $j$ th dimension of the $i$ th row in the dataset.

Assuming the data are $p$-dimensional, this computation requires $O(n)$ time for each of the $p(p-1) / 2$ off-diagonal entries in the matrix, or $O\left(n p^{2}\right)$ time overall. This can require a massive amount of computation. Imagine the problem of learning a statistical model over a database of 1 million medical images, wherein each 1,000-pixel by 1,000pixel image is mapped to a 1-million dimensional vector giving the intensity of each pixel. This computation will require on the order of $10^{18}$ floating point computations.

Addressing the difficulties. Mitigating the problem of over-fitting during covariance matrix estimation has been considered extensively in the statistics and machine learning literature [Friedman 1989; Levina et al. 2008; Bickel and Levina 2008b; Warton 2008]. A lot of different regularization methods have been proposed, but certainly the simplest and most straightforward is hard thresholding, or dropping the smallest values (in absolute value) in the covariance matrix [Bickel and Levina 2008a; Rothman et al. 2009; Cai and Liu 2011]. Specifically, we say that $\hat{\mathbf{S}}^{\prime}$ is "ŜS thresholded at cutoff $c$ " if, for each $(j, k)$ pair,

$$
\hat{s}_{j, k}^{\prime}= \begin{cases}0, & \text { if } \hat{s}_{j, k}<c \\ \hat{s}_{j, k}, & \text { otherwise }\end{cases}
$$

There are several theoretical arguments for thresholding as a form of regularization [Bickel and Levina 2008a; Rothman et al. 2009; Lam and Fan 2009], though an extensive study of these arguments is beyond the scope of the article. For suitably sparse covariance matrices, as long as $\frac{\log p}{n} \rightarrow 0$, the thresholded estimator consistently preserves the eigenvalues and eigenvectors of the true covariance matrix [Bickel and Levina 2008a]. This means that thresholding is particularly useful for applications that make the use of eigenvalues and eigenvectors, such as principal component analysis.

While regularization for covariance estimation, in general, and thresholding, in particular, have received considerable research attention, the computational problem of 


\begin{tabular}{|c|l|l|l|}
\hline & \multicolumn{1}{|c|}{ Exact } & \multicolumn{1}{c|}{ Fisher's } & \multicolumn{1}{c|}{ Gridded } \\
\hline Time complexity & $O\left(n p^{2}\right)$ in total & $O\left(K n+m p^{2}\right)$ in total & $O\left(K n+m p^{2}\right)$ in total \\
\hline Memory complexity & $\begin{array}{l}O\left(r p^{2}\right) \text { total RAM, } \\
\text { where } r \text { is the number } \\
\text { of producer threads }\end{array}$ & $O\left(p^{2}\right)$ total RAM & $O\left(m p^{2}\right)$ total RAM \\
\hline Main advantage & $\begin{array}{l}\text { Efficient when } p \text { is } \\
\text { small or data are sparse }\end{array}$ & $\begin{array}{l}\text { Efficient and accurate } \\
\text { when data are nearly } \\
\text { normal and dense }\end{array}$ & $\begin{array}{l}\text { High accuracy and } \\
\text { decent efficiency for } \\
\text { all kinds of data }\end{array}$ \\
\hline Main disadvantage & $\begin{array}{l}\text { RAM is overwhelmed } \\
\text { easily when } p \text { is large }\end{array}$ & $\begin{array}{l}\text { Very inaccurate when } \\
\text { data are skewed }\end{array}$ & Higher model complexity \\
\hline
\end{tabular}

Fig. 1. Relative merits of the various algoithms.

scaling such computations up to datasets having tens of thousands of dimensions and millions of data points has not. Perhaps the main reason for this is that it seems that there is little that can be done to speed the computation. If the goal is computing $\hat{\mathbf{S}}$, there seems to be no alternative to a careful implementation of the obvious, $O\left(n p^{2}\right)$ algorithm.

However, if one computes a thresholded covariance matrix for a threshold value that zeros most of the covariances, then there is a lot that can be done to speed the computation. Specifically, if the number of non-zero coefficients $K$ in the thresholded matrix $\hat{\mathbf{S}}^{\prime}$ is relatively small (so that $K$ is $O(p)$ ), then the identities of the $(j, k)$ pairs that will not be dropped can be computed quickly as an initial step-if $K$ is $O(p)$, then it takes $O(n p)$ time to compute the thresholded matrix $\hat{\mathbf{S}}^{\prime}$. For a high-dimensional problem, $O(n p)$ is likely to be much faster than $O\left(n p^{2}\right)$.

Our contributions. The principal contribution of this article is to develop several sampling-based algorithms for quickly computing an approximation to the set $\Lambda$-here $\Lambda$ is defined as the set of $(j, k)$ pairs that indicate which $\hat{s}_{j, k}$ will be included in the thresholded matrix. Although the algorithms we propose are not fundamentally distributed algorithms and can easily be implemented on a single machine, we describe them in a distributed context because for scaling any algorithm up to a 40,000 by 40,000 covariance matrix for a reasonably sized dataset, a distributed implementation is the only feasible alternative. Both algorithms take as an argument $K$ (the number of covariances that should remain in the thresholded matrix), and, in $O\left(m p^{2}\right)$ time, they compute a set $\Lambda^{\prime}$ where with high probability, $\Lambda \subset \Lambda^{\prime}$. Here, $m$ is the size of the sample that is used to compute $\Lambda^{\prime}$. The first algorithm we propose (which we call "Fisher's") relies on Fisher's transformation [Fisher et al. 1915] and works well with Gaussian data. The second algorithm, which we call "Gridded," is non-parametric. We have found that this method is able to approximate $\Lambda$ very well for even highly skewed data, using only $m=1,000$.

For comparison, we also consider how $\Lambda$ can be computed exactly in a distributed environment; we term this the "Exact" algorithm. The advantages and disadvantages of each are summarized in Figure 1.

Paper organization. In the next section of the article, we give a bit more background on covariance matrix thresholding. In Section 3, we consider how one might design a distributed code to efficiently (and exactly) compute a thresholded covariance matrix over a large and high-dimensional dataset. In Section 4, we develop our Z-Transformbased method. In Section 5, we consider potential problems with Fisher's method, and in Section 6, we propose another sampling-based method that is designed to be more robust. Section 7 details an experimental study, related work is covered in Section 8, and Section 9 ends the article. 


\section{BACKGROUND ON THRESHOLDING, RELATED WORK, AND PROBLEM DEFINITION}

The simplest thresholding method is known as "hard thresholding"; to perform hard thresholding, we simply drop all covariances smaller than a cutoff $c$. Several other ideas have been explored in the literature. Rothman et al. [2009] propose generalizing the idea of a hard thresholding function to a class of functions that can also incorporate other methods of shrinkage "shrinkage" is the general idea of having a parameter estimation framework that makes sure that smaller or less complicated parameters are chosen in order to avoid over-fitting; thresholding is one form of shrinkage). Specifically, they allow thresholding functions that not only drop $\hat{s}_{j, k}$ values with absolute value less than $c$, but that may also subtract (or add) a value $\leq c$ from $\hat{s}_{j, k}$ if $\hat{s}_{j, k}>c$. The authors argue that moderate shrinkage via thresholding can incur bias, but, in general, it helps lower the error of the estimated covariance matrix. In fact, the generalized thresholding algorithm is a good choice even if the sparsity of the covariance matrix is not known in advance. As Rotherman et al. show via simulation, combining hard and soft thresholding greatly increases the accuracy of the estimated covariance matrix for truly sparse models. But even if the matrix to be estimated is not sparse, the thresholded estimator does not perform worse than the sample covariance matrix.

Both approaches define a universal thresholding parameter for all off-diagonal elements. However, different dimensions of a dataset can have widely differing variances, which means that the covariances in $\hat{\mathbf{S}}$ can naturally vary by orders of magnitude. Universal thresholding approaches tend not to maintain their desirable asymptotic properties in such a situation. Cai and Liu [2011] consider two adaptive thresholding estimators for this problem. One thresholds different $\hat{s}_{j, k}$ values differently based on their respective variances. This estimator chooses an entry-dependent threshold value:

$$
c_{j, k} \propto \sqrt{\frac{\hat{\theta}_{j, k} \log p}{n}},
$$

where $\hat{\theta}_{j, k}$ is the variance of $\left(x_{i, j}-x_{i, k}\right)$, computed over the dataset. The resulting estimator significantly outperforms universal thresholding.

The preceding discussion assumes that the goal is to estimate the covariance matrix. It is often desirable to instead estimate the correlation matrix, whose entries always fall between -1 and 1 . Define the correlation $\hat{\rho}_{j, k}$ as follows:

$$
\hat{\rho}_{j, k}=\frac{\hat{s}_{j, k}}{\sqrt{\hat{s}_{j, j} \hat{s}_{k, k}}} .
$$

That is, $\hat{\rho}_{j, k}$ is the normalized value of the covariance $\hat{s}_{j, k}$ (also known as the correlation between dimensions $j$ and $k$ ).

In fact, thresholding the correlation matrix can be preferable to thresholding the covariance matrix directly. Cai and Liu [2011] propose thresholding the correlation matrix and then unnormalizing using the sample variances. First, a similar universal thresholding estimator can be used to estimate the thresholded correlation matrix $\hat{\mathbf{R}}^{\prime}=\left(\hat{\rho}_{j, k}^{\prime}\right)_{p \times p}$. Specifically, we let

$$
\hat{\rho}_{j, k}^{\prime}= \begin{cases}0, & \text { if } \hat{\rho}_{j, k}<c \\ \hat{\rho}_{j, k}, & \text { otherwise. }\end{cases}
$$

The corresponding covariance matrix can then be estimated by $\hat{\mathbf{S}}^{\prime}=\mathbf{D}^{1 / 2} \hat{\mathbf{R}}^{\prime} \mathbf{D}^{1 / 2}$ where $\mathbf{D}=\operatorname{diag}(\hat{\mathbf{S}})$ consists of the variances. This outperforms thresholding the covariance matrix directly, and the consistency of doing so is justified in Jiang [2013].

One concern when thresholding is that the resulting matrix will not have positive semi-definiteness, which is a requirement for some applications. This issue has been 
considered in the literature. For example, in Bickel and Levina [2008a], the authors show that the thresholded matrix will remain positive semi-definite if

$$
\left\|\hat{\mathbf{S}}_{c}^{\prime}-\hat{\mathbf{S}}\right\|<\lambda_{\min }(\hat{\mathbf{S}}),
$$

where $\|\cdot\|$ is the $L_{2}$ norm and $\lambda_{\min }(\hat{\mathbf{S}})$ is the smallest eigenvalue of $\hat{\mathbf{S}}$. Other papers also discuss the issue of achieving sparseness via thresholding while at the same time preserving positive semi-definiteness when estimating covariance matrices [Xue et al. 2012; Guillot and Rajaratnam 2012].

We note that in some important applications for large covariance estimation, positive semi-definiteness is not actually a requirement. Specifically, consider the problem of sparse matrix inversion, where the goal is to approximately invert a large matrix while ensuring the sparsity of the computed inverse. Typically, the output of such an algorithm will be used as input to a Gaussian Markov random field. Crucially, these inversion algorithms do not always require the input to be positive semi-definite. For example, Cai et al. [2011] show how to remedy non-positive semi-definiteness by replacing $\hat{\mathbf{S}}^{\prime}$ with $\hat{\mathbf{S}}^{\prime}+\epsilon \mathbf{I}$ for some small $\epsilon>0$, where $\epsilon$ is carefully chosen so that it does not have a significant effect on the computational accuracy of the final inverse but is large enough to make $\hat{\mathbf{S}}^{\prime}$ positive semi-definite.

Matrix sparsification and approximation. Besides thresholding, many other matrix regularization and approximation approaches have been proposed in the literature. Examples include banding [Bickel and Levina 2008b], where the idea is to confine the nonzero entries in a matrix to a diagonal band, and tapering [Furrer and Bengtsson 2007] where the idea is to shrink the off-diagonal entries of the covariance matrix. Another idea is penalized estimation [Huang et al. 2006], where $L_{1}$ penalties are applied on the Cholesky factor. Factor-model-based model estimation has also been proposed for the task [Fan et al. 2008, 2011] wherein a factor-based model is constructed for the observed data, and sparsity is imposed on the learned model. The Nyström method [Williams and Seeger 2001] generates a low-rank approximation to the original matrix by sampling from its columns. Our focus in this article is on the scaling of regularization/ approximation to very large problem, rather than proposing a new regularization/ approximation method. We have chosen to study thresholding in this context, though future studies might consider some of these other methods as alternatives.

Other related work. Efficient computation of an approximate inverse covariance matrix has been the focus of almost all recent related, computationally oriented work in this area. Such methods typically add a lasso penalty to the inverse problem and focus on solving the resulting optimization task. For example, Yuan and Lin [2007] propose estimating the sparse precision matrix $\Omega$ that maximizes the following equation:

$$
\hat{\Omega}=\operatorname{argmax}_{\Omega}\left\{\log |\Omega|-\operatorname{trace}(\Omega \hat{\mathbf{S}})-\lambda\|\Omega-\operatorname{diag}(\Omega)\|_{1}\right\} .
$$

In this expression, $\lambda$ controls the extent of the regularization; if $\lambda$ is large, it penalizes a large number of significant or non-zero entries in the learned matrix $\hat{\Omega}$.

Given $\hat{\mathbf{S}}$, computing the optimal value of $\hat{\Omega}$ is then a challenging optimization problem, especially when the data are high dimensional. There has been a lot of work (particularly in the machine learning literature) over the last few years that focuses on solving the problem efficiently. There are too many papers on this topic to list all of them; a small selection is given in the references section [Kolar and Xing 2012; Hsieh et al. 2013; Rolfs et al. 2012; Olsen et al. 2012; Hsieh et al. 2011]. Kolar and Xing [2012] handle data with missing values by adopting a two-step procedure that converges quickly and does not require imputation of the missing values. Hsieh et al. [2013] introduce a novel block-coordinate descent method, whereas [Rolfs et al. 2012] 
propose a proximal gradient method to solve the $L_{1}$-regularized maximum likelihood estimation problem. The algorithms in Olsen et al. [2012] and Hsieh et al. [2011] are based on Newton's method and second-order gradient information.

The closest work to our own from the database and data mining literature is the problem of finding strong correlations in a large dataset [Xiong et al. 2006; Cohen et al. 2001; Ilyas et al. 2004; Grahne et al. 2000]. Such algorithms are always related to association rule mining, which discovers strong rules existing among variables in large databases, or some of the characteristics of Pearson's correlation coefficient. For example, Xiong et al. [2006] take advantage of the 2D monotone property of the upper bound of the correlation coefficient. Ilyas et al. [2004] develop a system that could detect strong correlations and dependencies with high scalability based on sampling.

We also mention that there has been a lot of work in the database area on sampling [Haas and Swami 1992; Lipton et al. 1990; Chaudhuri et al. 1998] and even some work on sampling in a distributed system [Pansare et al. 2011]. However, none of this work specifically targets covariance estimation.

Our thresholding problem. In this article, we will consider a slightly modified version of the correlation-based thresholding estimator. Specifically, we consider how to compute a set $\Lambda$ of (dimension, dimension) pairs with size $K$, where $\Lambda$ is defined as follows.

Definition 2.1 (The top-K correlation problem). Let $\Lambda$ be a set of $(j, k)$ pairs of size $K . \Lambda$ is a solution to the top- $K$ correlation problem if, for each $(j, k)$ included in $\Lambda$, it is the case that $j \neq k$ and $\hat{\rho}_{j, k}$ is one of the $K$ largest correlations in $\hat{\mathbf{R}}$ (where $\hat{\mathbf{R}}$ is the sample correlation matrix).

That is, we do not directly consider the problem of computing the thresholded correlation matrix $\hat{\mathbf{R}}^{\prime}$. Rather, we consider the problem of computing which correlations one would need to compute in order to obtain $\hat{\mathbf{R}}^{\prime}$. Once $\Lambda$ has been computed, $\hat{\mathbf{R}}^{\prime}$ can itself be computed in $O(K n)$ time. The reason that we define the problem in this way is that it allows us to design approximation algorithms for $\Lambda$ which may find "false positives"-that is, additional (dimension, dimension) pairs that do not correspond to the top $K$ correlations. Since we can drop additional correlations outside of the top $K$ after computing them, false positives will not affect the accuracy of the computed $\hat{\mathbf{S}}^{\prime}$.

Also note that rather than taking the threshold value $c$ as input, we instead take as input the number of non-diagonal entries $K$ to retain. We choose to parameterize the problem in this way because it allows us to directly control the number of nonzero entries in the thresholded matrix, and hence the computational complexity of the problem. However, the two parameterizations are equivalent, in the sense that a given $K$ is associated with a unique $c$ value, and a given $c$ uniquely determines $K$ (assuming that no two entries in $\hat{\mathbf{R}}$ have the same value).

Finally, without loss of generality, we assume that each dimension has unit variance so that $\hat{\mathbf{R}}$ and $\hat{\mathbf{S}}$ are identical, and in the remainder of the article, we do not make a distinction between $\hat{\mathbf{R}}$ and $\hat{\mathbf{S}}$. Thus, we can compute the $K$ entries in $\Lambda$ by computing the $K$ largest covariances in $\hat{\mathbf{S}}$. If each dimension does not have unit variance, then the data can be normalized, after computing the variance of each dimension.

\section{THE EXACT METHOD}

Before turning to the question of how to approximate $\Lambda$, we first consider how to compute $\Lambda$ exactly. The obvious (though not always computationally feasible) method for computing $\Lambda$ is to compute all of the entries in $\hat{\mathbf{S}}$ (or $\hat{\mathbf{R}}$ ) exactly, and then to simply choose the largest $K$. In this section, we discuss the exact method in some detail. 


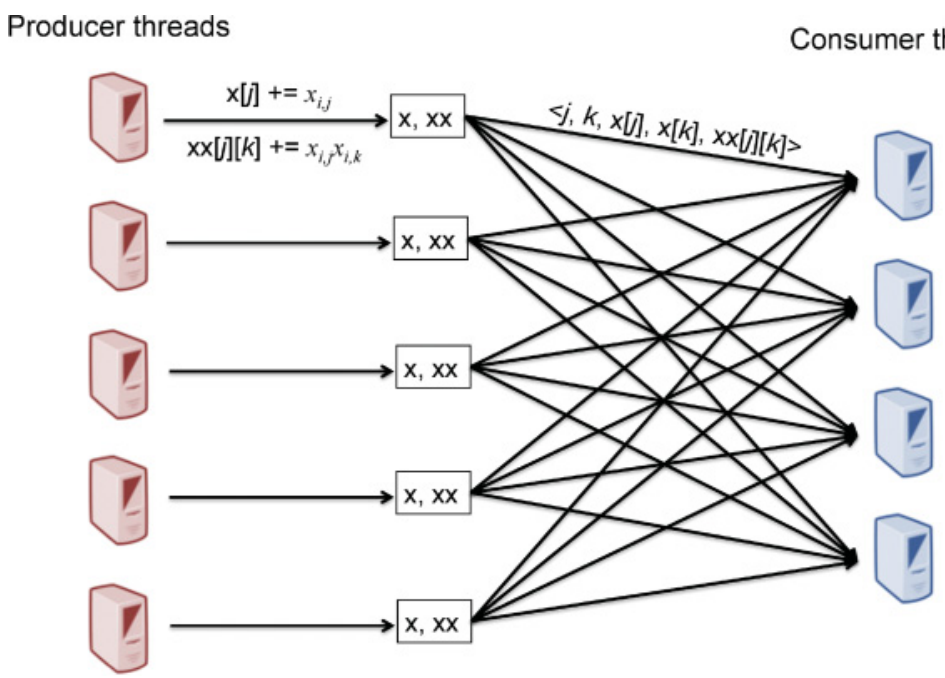

Fig. 2. The producer threads and consumer threads for the exact method. The statistics $\mathrm{x}$ and $\mathrm{xx}$ are preaggregated on each producer thread, and then transmitted to the corresponding consumer threads, based on the values of $j$ and $k$.

\subsection{Exact Algorithm for Dense Data}

We assume that a large number of producer threads are scattered around a compute cluster, and that each producer thread is assigned a number of rows from the dataset $\mathbf{X}$ to operate on. A "thread" here is an independent computing unit that allows for one sequential stream of instructions to be executed. These threads might be mappers in a MapReduce environment [Dean and Ghemawat 2008] or they might be threads created via a package such as pthreads or Message Passing Interface (MPI) [Gropp et al. 1999]. We assume that there are also $t$ consumer threads, where each consumer thread is assigned one or more dimension-dimension pairs for which the thread is responsible for computing $\hat{s}_{j, k}$ (we will assume that the the pair $(j, k)$ is assigned to consumer thread $j \times p+k \bmod t$ ). We assume that producer threads can send data to consumer threads. In MapReduce, this may happen via a MapReduce shuffle. Communication could also be implemented via MPI, or via operating system sockets, or via any other of a wide variety of mechanisms.

Given this setup, it is clear that we need two classes of statistics to compute $\hat{\mathbf{S}}$ exactly:

(1) $\sum_{i} x_{i, j}$ for each $j$ in $\{1 \ldots p\}$.

(2) $\sum_{i} x_{i, j} x_{i, k}$ for each $\{j, k\}$ subset of $\{1 \ldots p\}$, where $j \leq k$.

Algorithm 1 can be used to compute and send the required statistics from each producer thread to all of the consumer threads. Figure 2 shows the producer-consumerthread framework for the exact method in a distributed setting.

Once all of the producer threads have completed execution, each consumer thread simply sorts all of the tuples that it receives into a set of groups, where each tuple in each group has the same pair of $j$ and $k$ values. The tuples for a given $(j, k)$ pair are then aggregated by summing all of the $\langle\mathrm{x}[j], \mathrm{x}[k], \mathrm{xx}[j][k]\rangle$ values in the group, and the triple resulting from the summation is used to compute the final $\hat{s}_{j, k}$ value for that $(j, k)$ pair. 


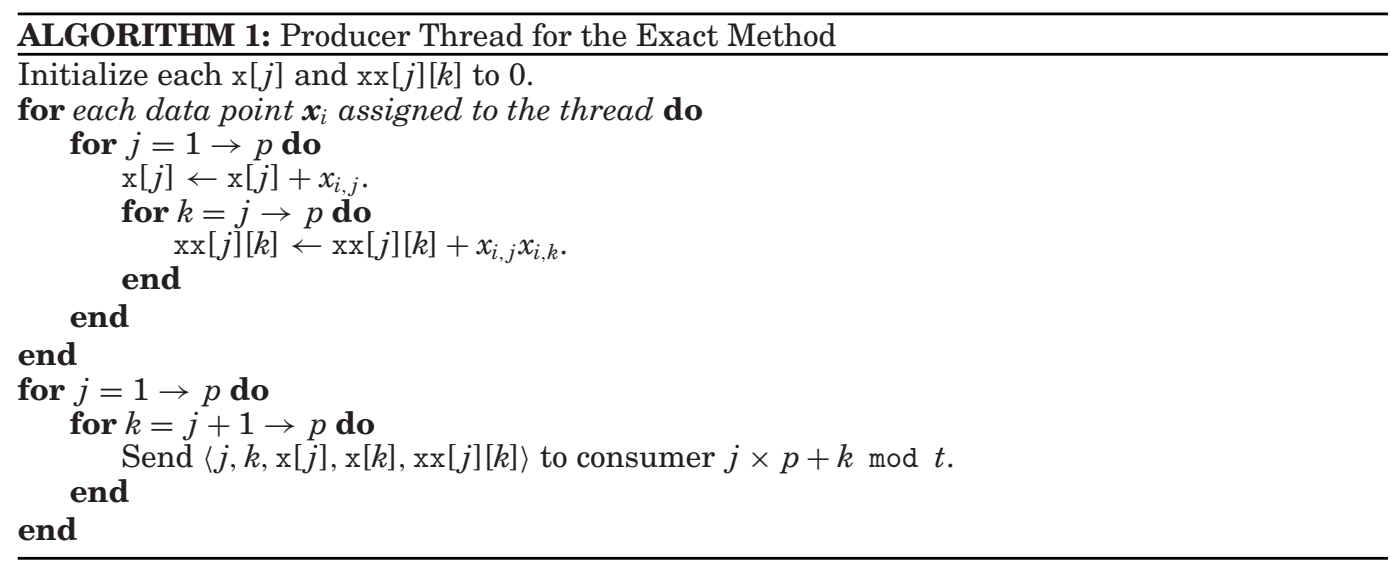

\subsection{Taking Advantage of Sparsity}

Overall, this algorithm has complexity $O\left(n p^{2}\right)$, since it must consider every possible $\mathbf{x}_{i}$ for every possible $(j, k)$ dimension pair. It is not possible to do better than this if the data are dense (that is, if most of the values are non-zero), but it is possible to do a lot better if the data are sparse. Specifically, the first set of nested loops in Algorithm 1 is changed to the following algorithm.

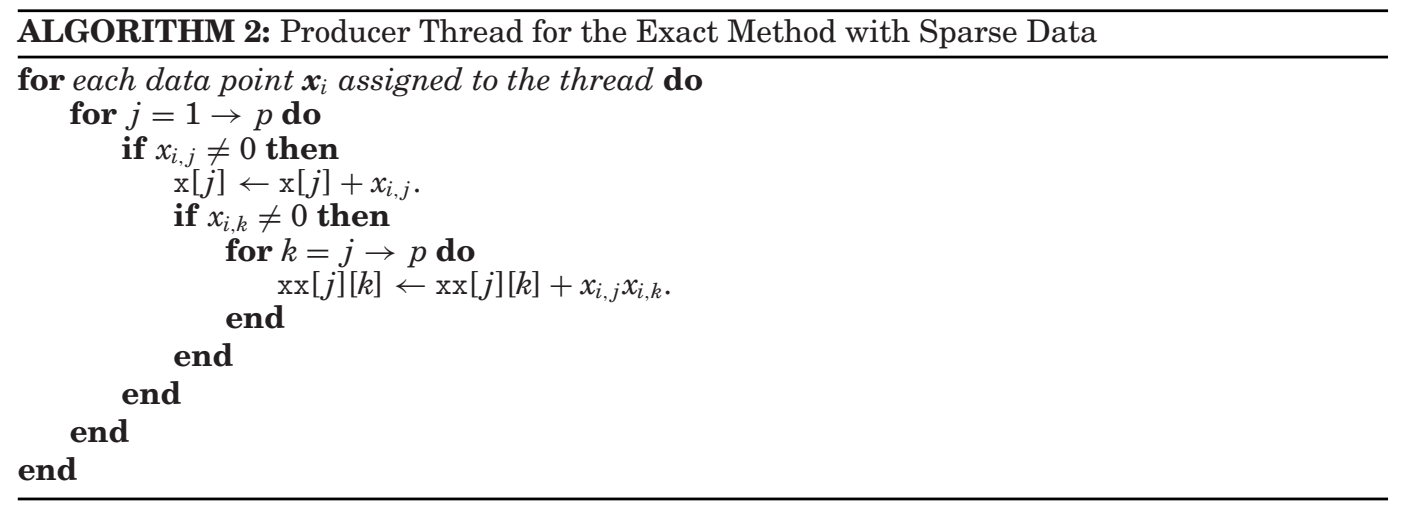

Because a zero-valued $x_{i, j}$ can neither contribute to $\mathrm{x}[j]$ nor $\mathrm{xx}[j][k]$, we can skip dimensions that are zero, as is done above. If this skipping is implemented intelligently (that is, using a sparse representation for each $\mathbf{x}_{i}$ so that we can immediately skip over such dimensions), then this brings the complexity of the exact computation down to $O\left(n q^{2}\right)$ (this is assuming that each entry in X has $q$ non-zero entries). If $q \ll p$, this can be a huge reduction in complexity.

\section{FISHER'S METHOD}

The idea we explore in the next two sections is to use sampling-based algorithms to estimate a set of $(j, k)$ pairs $\Lambda^{\prime}$, where $\Lambda^{\prime}$ is computed so that with probability of $(1-\alpha)$, $\Lambda \subseteq \Lambda^{\prime}$ for a user-supplied error threshold $\alpha$. If this can be done in $O\left(m p^{2}\right)$ time using a set of $m$ samples where $m \ll n$, this can represent a huge time savings compared to computing $\Lambda$ exactly. If this can be done in such a way that $\Lambda^{\prime}$ is not much larger than $K$, then the cost of computing $\hat{\mathbf{S}}^{\prime}$ from $\Lambda^{\prime}$ is still reasonable. 
A further advantage of sampling is that it allows us to avoid the $O\left(n p^{2}\right)$ memory cost for storing the xx array at each producer. As we will see, since we are subsampling, each producer thread will be able to simply send the sampled data on to the relevant consumer threads, rather than pre-aggregating at each producer. This will reduce the overall RAM requirement to $O\left(n p^{2}\right)$ for the entire compute cluster.

\subsection{Fisher's Z-Transform}

The first sampling-based solution we describe is based on the well-known Fisher's Z-Transform, proposed in 1915 [Fisher et al. 1915]. Fisher's Z-Transform is used to estimate the correlation $\rho$ from a set of normally distributed sampled data by converting the non-normally distributed sampling distribution of Pearson's correlation $r$ (computed over the sample) into Fisher's $z$, which is normal and can be used to produce confidence bounds on the population correlation $\hat{\rho}$. For a pair of dimensions $(j, k)$, the procedure is described as follows.

(1) Randomly sample a set of $\left(x_{i, j}, x_{i, k}\right)$ pairs and use them to compute the sample correlation $r_{j, k}$.

(2) Perform the Z-Transform $z_{j, k}=\frac{1}{2} \ln \left(\frac{1+r_{j, k}}{1-r_{j, k}}\right)$.

(3) It is known that $z_{j, k}$ is approximately normally distributed with mean $\frac{1}{2} \ln \left(\frac{1+\hat{\rho}_{j, k}}{1-\hat{\rho}_{j, k}}\right)$ and standard error $\frac{1}{\sqrt{m_{j, k}-3}}$, where $m_{j, k}$ is the sample size. Here, $z_{j, k}$ is an estimator of Fisher's transformed correlation $\frac{1}{2} \ln \left(\frac{1+\hat{\rho}_{j, k}}{1-\hat{\rho}_{j, k}}\right)$, rather than of $\hat{\rho}_{j, k}$ itself. The normal distribution of $z_{j, k}$ can be used to construct confidence intervals on $\hat{\rho}_{j, k}$. First the $(1-\theta) \%$ one-tailed confidence intervals for $\frac{1}{2} \ln \left(\frac{1+\hat{\rho}_{j, k}}{1-\hat{\rho}_{j, k}}\right)$ are computed as $\left(-\infty, z_{j, k}+\frac{\Phi^{-1}(1-\theta)}{\sqrt{m_{j, k}-3}}\right)$ and $\left(z_{j, k}-\frac{\Phi^{-1}(1-\theta)}{\sqrt{m_{j, k}-3}},+\infty\right)$, where $\Phi^{-1}(1-\theta)=-\Phi^{-1}(\theta)$ is the $100(1-\theta)$ percentage point of the standard normal distribution. Then, these confidence intervals are transformed back to derive the one-tailed lower and upper endpoints for $\hat{\rho}_{j, k}$ :

$$
\left(-\infty, \tanh \left[\frac{1}{2} \ln \left(\frac{1+r_{j, k}}{1-r_{j, k}}\right)-\frac{\Phi^{-1}(\theta)}{\sqrt{m_{j, k}-3}}\right]\right)
$$

and

$$
\left(\tanh \left[\frac{1}{2} \ln \left(\frac{1+r_{j, k}}{1-r_{j, k}}\right)+\frac{\Phi^{-1}(\theta)}{\sqrt{m_{j, k}-3}}\right],+\infty\right),
$$

where $\tanh (x)=\frac{\exp (2 x)-1}{\exp (2 x)+1}$.

Using a very similar computation, the probability that $\hat{\rho}_{j, k}$ is located above or below an endpoint $\epsilon$ can be computed:

$$
\operatorname{Pr}\left(\hat{\rho}_{j, k} \geq \epsilon\right)=\Phi\left[\sqrt{m_{j, k}-3}\left(\frac{1}{2} \ln \left(\frac{1+r_{j, k}}{1-r_{j, k}}\right)-\frac{1}{2} \ln \left(\frac{1+\epsilon}{1-\epsilon}\right)\right)\right],
$$

and

$$
\operatorname{Pr}\left(\hat{\rho}_{j, k} \leq \epsilon\right)=\Phi\left[\sqrt{m_{j, k}-3}\left(\frac{1}{2} \ln \left(\frac{1+\epsilon}{1-\epsilon}\right)-\frac{1}{2} \ln \left(\frac{1+r_{j, k}}{1-r_{j, k}}\right)\right)\right] .
$$

As we describe in the next few subsections, these bounds can be used to approximately compute $\Lambda$. 


\subsection{Producer Thread Implementation}

To utilize Fisher's in a distributed setting, producer threads are responsible for performing the sampling. Given a user-supplied sample size $m \ll n$, our goal is to sample a number of $\left\langle j, k, x_{i, j}, x_{i, k}\right\rangle$ tuples so that the probability of selecting a particular tuple is $\frac{m}{n}$; each sampled tuple is then sent to the appropriate consumer thread for post-processing (this post-processing is described in the next subsection). The most important consideration when implementing the producer thread is that the complexity should be $O\left(m p^{2}\right)$ which is equivalent to $O\left(p^{2}\right)$, assuming small $m$. Practically speaking, this means that we must avoid doing the sampling within the inner-most loop, as in the following algorithm.

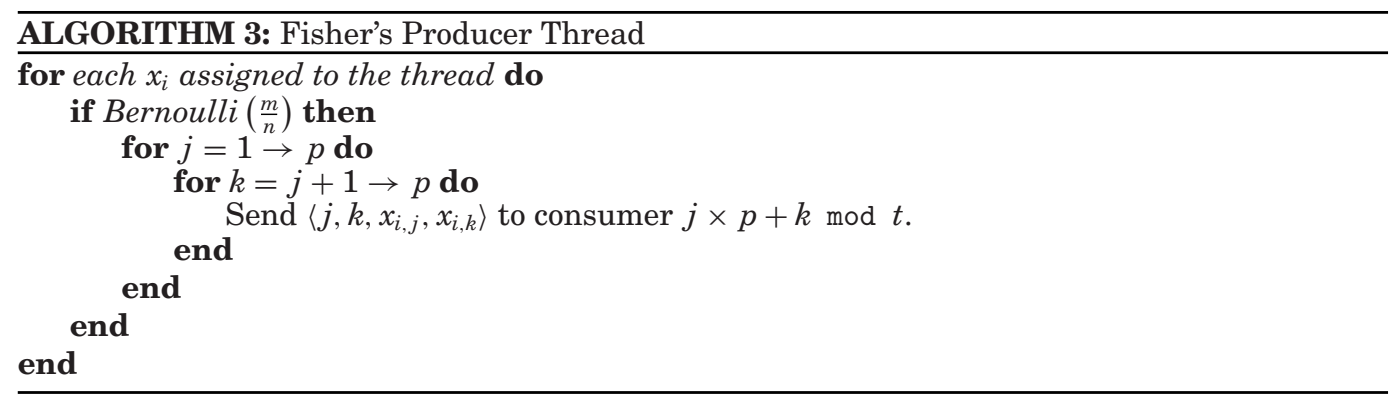

Although this sampler is going to be fast, there is a subtle problem: The algorithm samples all of the statistics associated with a single data point, or else it samples none of them. If $p$ is very large, then the sheer size of the output when a data point is sampled is debilitating. Especially in a large compute cluster where the number of threads greatly exceeds $m$, we will see a situation where most threads (and most machines) output no data, but a few threads produce a huge number.

Obviously, it is desirable to spread out the statistics collection among the various threads. With a bit of care, we can do this by randomly selecting $\langle i, j, k\rangle$ triples (where a triple specifies a specific $(j, k)$ pair for a specific data point $\mathbf{x}_{i}$ ) and only sending along the $\left\langle j, k, x_{i, j}, x_{i, k}\right\rangle$ tuple for those triples that are selected, rather than selecting particular data points and sending all of the tuples that are associated with the data point.

To do this, we view a producer thread that manages $d$ data points as having a set of $\frac{d p^{2}}{2}$ different triples that it can choose from, and we simulate scanning each of those triples, and for each, flipping a coin whose probability of being heads is $\frac{m}{n}$. If the coin comes up heads, we pick the triple and output it for use by the appropriate consumer thread.

Obviously, we do not want to actually perform this huge number of random trials, but we can define a process that is statistically equivalent and much more computationally efficient. To do this, we first generate the number according to a num subject to a Binomial $\left(\frac{d p^{2}}{2}, \frac{m}{n}\right)$ distribution. This is the number of heads that were observed (or, equivalently, the number of tuples that we will send on to an appropriate consumer thread). We then choose num different triples uniformly, without replacement, and output each to the appropriate consumer thread. The algorithm is given as Algorithm 4.

In this code, pickEntry() maps a value in $\left\{1 \ldots \frac{d p^{2}}{2}\right\}$ to a specific $(j, k)$ where $j, k \leq p$ and $j \leq k$. 


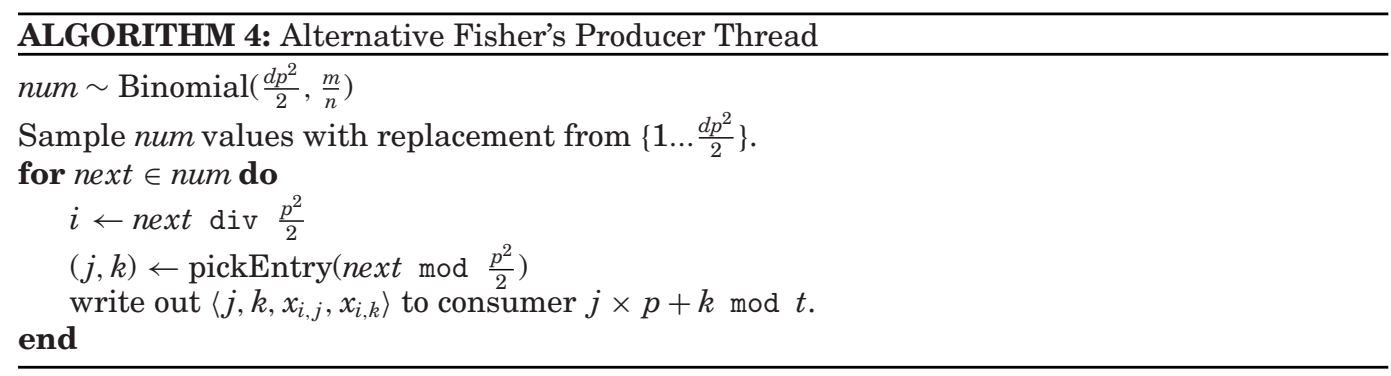

\subsection{Consumer Thread Implementation}

Once the producer threads complete, each consumer thread will obtain a set of sampled $\left\langle j, k, x_{i, j}, x_{i, k}\right\rangle$ tuples. The thread groups those tuples according to the $j$ and $k$ values. After this, the consumer thread sends the single tuple $\left\langle j, k, r_{j, k}, \sqrt{\frac{1}{m_{j, k}-3}}\right\rangle$ to a central location for the final thresholding.

\subsection{Thresholding}

The central location will then make use of the standard confidence bounds provided by Fisher's transformation to bound the actual value of the correlation and perform the final thresholding.

Remember, our goal is not to estimate a particular covariance; our goal is to threshold the covariance matrix. That is, we wish to approximate the set $\Lambda$ of the $(j, k)$ pairs that indicate which $\hat{s}_{j, k}$ will be included in the thresholded matrix, where $\Lambda$ is defined so that if $(j, k)$ is in $\Lambda$ and $\left(j^{\prime}, k^{\prime}\right)$ is outside of $\Lambda$, then $\left|\hat{\rho}_{j, k}\right| \geq\left|\hat{\rho}_{j^{\prime}, k^{\prime}}\right|$. By "approximate," we mean that we compute $\Lambda^{\prime}$ so as to guarantee that

$$
\operatorname{Pr}\left(\Lambda \subseteq \Lambda^{\prime}\right) \geq 1-\alpha,
$$

where $\alpha$ is a user-supplied threshold. The size of set $\Lambda^{\prime}$ will be between $K$ and $\frac{p(p-1)}{2}$, and ideally it will be very close to $K$.

To actually perform the thresholding note that we can reduce the problem of thresholding to the problem of computing a cutoff $c_{v}$ such that:

(i) with high probability, at least $K$ correlations corresponding to the (dimension, dimension) pairs in $\Lambda^{\prime}$ are in reality above $c_{v}$; and

(ii) with high probability, every correlation corresponding to the (dimension, dimension) pairs outside of $\Lambda^{\prime}$ (denoted by $\left.\left(\Lambda^{\prime}\right)^{C}\right)$ is below $c_{v}$.

During thresholding, various candidate values for $c_{v}$ will be generated via a linesweeping algorithm. Thus, the key question becomes, given a cutoff $c_{v}$, can we check to see if a candidate set $\Lambda^{\prime}$ satisfies the both conditions?

First, we want to ensure that condition (i) holds with probability $1-\alpha / 2$. To do this, choose the (dimension, dimension) pairs in $\Lambda^{\prime}$ corresponding to the $K$ largest sample correlations and name this subset as $\Lambda_{K}^{\prime}$. From Boole's inequality [Diananda 1953] (also known as the "union bound"), all we need to do is to ensure that

$$
\sum_{(j, k) \in \Lambda_{K}^{\prime}} \operatorname{Pr}\left(\hat{\rho}_{j, k} \leq c_{v}\right) \leq \alpha / 2 .
$$

Note that the probability required within the summation is exactly the one-tailed upper probability from Section 4.1 with $\epsilon=c_{v}$. 
We also need to ensure that with high probability, condition (ii) holds, that is, we wish to ensure that every correlation corresponding to a (dimension, dimension) pair outside of $\Lambda^{\prime}$ is below $c_{v}$. As similar argument holds here, we can derive the requirement that

$$
\sum_{(j, k) \in\left(\Lambda^{\prime}\right)^{C}} \operatorname{Pr}\left(\hat{\rho}_{j, k} \geq c_{v}\right) \leq \alpha / 2 .
$$

Similarly, the probability required within the summation is simply the one-tailed lower probability with $\epsilon=c_{v}$.

\subsection{Implementation}

At the central location that has received all of the $\left\langle j, k, r_{j, k}, d_{j, k}\right\rangle$ tuples, we sort the correlations in reverse order by $r_{j, k}$ and then run a line sweep, testing $c_{v}$ values from one down to zero.

For a given cutoff $c_{v}$, we add all (dimension, dimension) pairs corresponding to the sample correlations whose expected value is greater than $c_{v}$ to the set $\Lambda^{\prime}$. Assuming the size of $\Lambda^{\prime}$ is at least $K$, this allows us to compute both $\Lambda_{K}^{\prime}$ and $\left(\Lambda^{\prime}\right)^{C}$. We then check whether Equation (1) is satisfied for $\Lambda_{K}^{\prime}$. If it is, then we have satisfied requirement (i) at this cutoff. If not, then we move the cutoff to the right and try the whole process again.

If we have satisfied requirement (i), we must now compute the final set $\left(\Lambda^{\prime}\right)^{C}$ that satisfies requirement (ii). Here, we perform a second line sweep to choose the correlations inside of $\left(\Lambda^{\prime}\right)^{C}$ : Moving from large expected value of correlation to small, we remove the corresponding (dimension, dimension) pairs from $\left(\Lambda^{\prime}\right)^{C}$ one by one, adding them to $\Lambda^{\prime}$. For each possible $\left(\Lambda^{\prime}\right)^{C}$, we check whether Equation (2) is satisfied. If it is, we have completed the thresholding process. At this point, we return the (dimension, dimension) pairs in set $\Lambda^{\prime}$. If it is not, then we add one more (dimension, dimension) pair from $\left(\Lambda^{\prime}\right)^{C}$ to $\Lambda^{\prime}$ and try again.

It is easy to argue that this algorithm will find a solution. Note that $\Lambda_{K}^{\prime}$ stays fixed so $\sum_{(j, k) \in \Lambda_{K}^{\prime}} \operatorname{Pr}\left(\hat{\rho}_{j, k} \leq c_{v}\right)$ must get smaller as $c_{v}$ decreases during the line sweep. Thus, we will eventually satisfy the requirement of Equation (1). And by removing correlations from $\left(\Lambda^{\prime}\right)^{C}$, we know that we will eventually satisfy the requirement of Equation (2). In the degenerate case, the final $\Lambda^{\prime}$ will contain all (dimension, dimension) pairs, but this is still a viable solution.

\section{FISHER'S AND NON-NORMALITY}

The assumption underlying Fisher's method is that the joint distribution along every dimension-dimension pair is bivariate normal. If this assumption is reasonable, then the method of the previous section is an excellent choice. Unfortunately, the assumption can be quite problematic if the joint distribution is not bivariate normal, resulting in bounds on the actual correlation that are inaccurate. This, in turn, can lead to an inaccurate choice for the set of corresponding (dimension, dimension) pairs to include in $\Lambda^{\prime}$.

For a simple example of the effect of non-normality, consider Figures 3(a) and (b) which consider two datasets, both of which have identical covariance matrices (and hence identical correlation matrices). The dataset pictured in Figure 3(a) is bivariate normal, and the dataset in Figure $3(\mathrm{~b})$ is not.

For each dataset, we repeatedly sample 50 data points and use Fisher's method to estimate the correlation of the two variables. For each dataset, we plot a histogram of the various correlation estimates obtained (the plots are in Figures 3(c) and (d)). In each case, the true correlation, shown as a red line, is around -0.58 . For the normal data, the sample correlations lies in a relatively small range from -0.8 to -0.2 , whereas for 

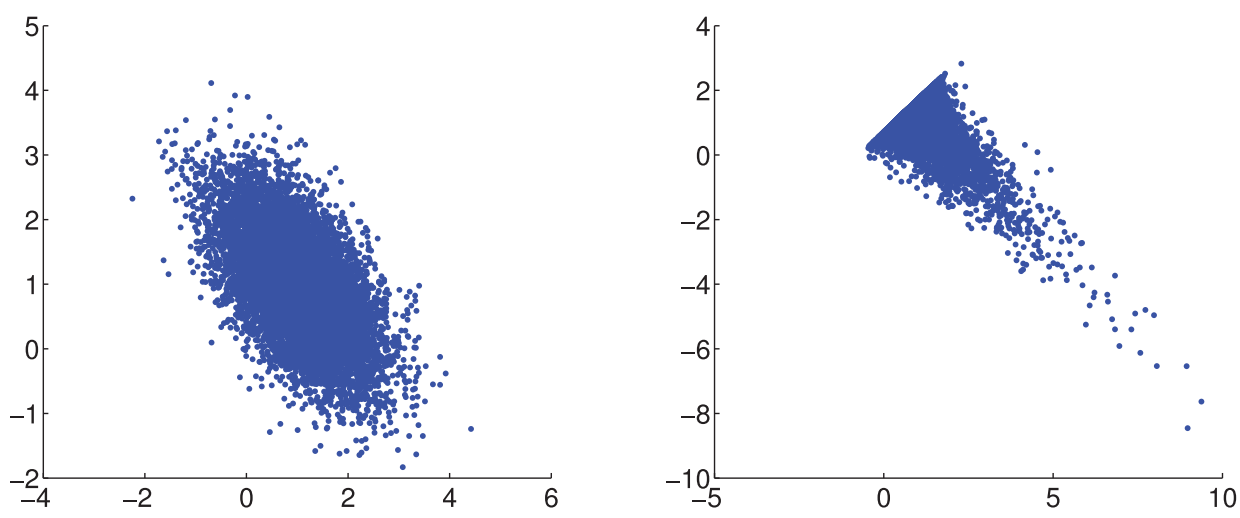

(a) Data distribution of the bivariate normal dataset (b) Data distribution of the non bivariate normal dataset
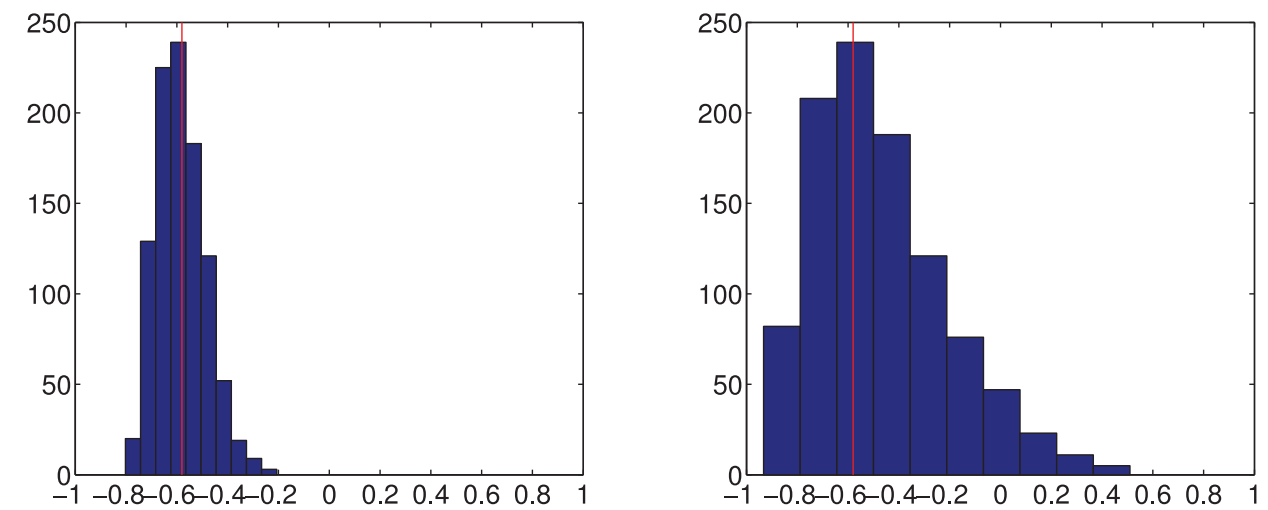

(c) Estimated correlations of the bivariate normal (d) Estimated correlations of the non bivariate normal dataset dataset

Fig. 3. Two small datasets, as well as the histograms for 1,000 estimated sample correlations. The red line in each histogram shows the true correlation.

the non-normal data, the sample correlations are more spread out, from -0.9 to 0.5 . This shows the significant increase in variance of the correlation estimate induced by the non-normality of the second case. Any bounds derived from such a dataset using Fisher's method are going to be very aggressive, likely leading to a $\Lambda^{\prime}$ that contains few of the (dimension, dimension) pairs that correspond to the actual top $K$ correlations.

\section{THE GRIDDED APPROACH}

\subsection{The Basic Approach}

Given the natural concerns associated with the Fisher approach, in this section, we consider a simple, non-parametric alternative. For a particular $(j, k)$ pair, we will construct a two-dimensional, spatial grid. We will then count the number of samples that appear in each cell of the grid and use those counts to estimate the probability that an arbitrary database point will fall in the cell (see Figure 4). If we assume that points are distributed uniformly within a cell, then it is an easy matter to estimate the correlation $\hat{\rho}_{j, k}$. 

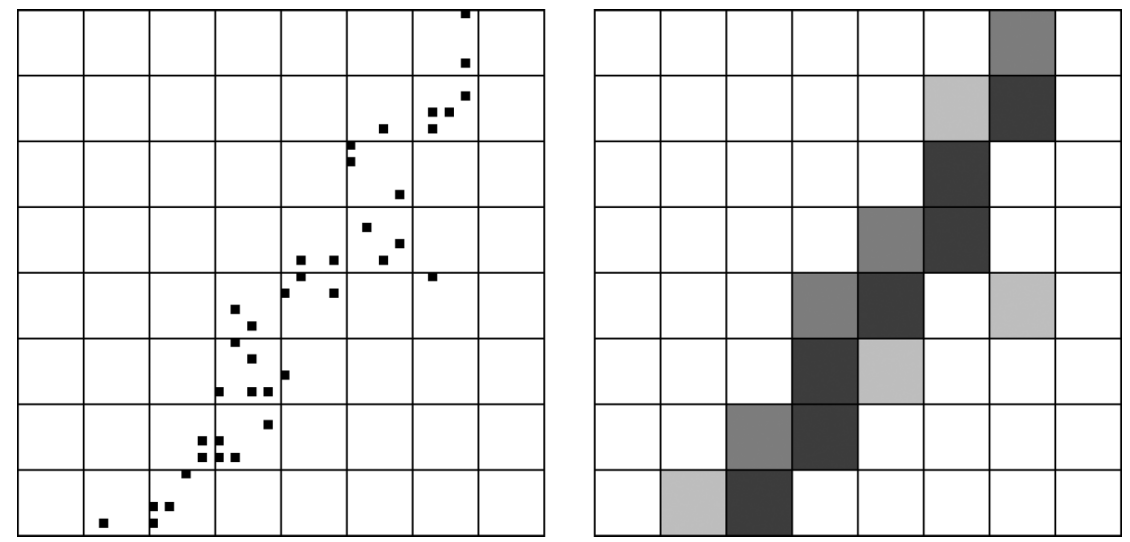

Fig. 4. Estimating the PDF of a distribution from a sample (depicted at top) via the gridded approach. The simplest method to estimate the density $\pi_{a, b}$ of a cell $(a, b)$ is to compute $c_{a, b}$, the number of points in the cell, and set $\pi$ to be proportional to this count, as is shown at the bottom of the figure.

Specifically, let $X_{j}$ denote the random variable that governs the distribution of the data over dimension $j$, and let $X_{k}$ denote the random variable that governs the distribution of data over dimension $k$. Then, we know that

$$
\rho\left(X_{j}, X_{k}\right)=\frac{\mathbb{E}\left[X_{j} X_{k}\right]-\mathbb{E}\left[X_{j}\right] \mathbb{E}\left[X_{k}\right]}{\sqrt{\mathbb{E}\left[X_{j}^{2}\right]-\mathbb{E}^{2}\left[X_{j}\right]} \sqrt{\mathbb{E}\left[X_{k}^{2}\right]-\mathbb{E}^{2}\left[X_{k}\right]}} .
$$

If we make the reasonable assumption that, for a large data set, $\rho\left(X_{j}, X_{k}\right) \approx \hat{\rho}_{j, k}$, (that is, we assume that the actual correlation of the random variables underlying the data is the same as the correlation observed in the data), then we need to simply compute the various expectations in the above formula in order to estimate $\hat{\rho}_{j, k}$.

To do this, let $l_{j, a}$ denote the lower spatial boundary of the ath row of cells in the grid, and let $h_{j, a}$ denote the upper boundary of this row. Likewise, let $l_{k, b}$ denote the left spatial boundary of the $b$ th column of cells in the grid, and let $h_{k, b}$ denote the right boundary of the column. Furthermore, let $\pi_{a, b}$ denote the estimated density of the cell in row $a$ and column $b$ (that is, the probability that a random data point will fall in that cell). Then, it is easily shown that

$$
\begin{aligned}
& \mathbb{E}\left[X_{j}\right]=\sum_{a} \sum_{b} \frac{1}{2}\left(l_{j, a}+h_{j, a}\right) \cdot \pi_{a, b}, \\
& \mathbb{E}\left[X_{k}\right]=\sum_{a} \sum_{b} \frac{1}{2}\left(l_{k, b}+h_{k, b}\right) \cdot \pi_{a, b}, \\
& \mathbb{E}\left[X_{j}^{2}\right]=\sum_{a} \sum_{b} \frac{1}{3}\left(l_{j, a}^{2}+l_{j, a} h_{j, a}+h_{j, a}^{2}\right) \cdot \pi_{a, b}, \\
& \mathbb{E}\left[X_{k}^{2}\right]=\sum_{a} \sum_{b} \frac{1}{3}\left(l_{k, b}^{2}+l_{k, b} h_{k, b}+h_{k, b}^{2}\right) \cdot \pi_{a, b}, \\
& \mathbb{E}\left[X_{j} X_{k}\right]=\sum_{a} \sum_{b} \frac{1}{4}\left(l_{j, a}+h_{j, a}\right)\left(l_{k, b}+h_{k, b}\right) \cdot \pi_{a, b} .
\end{aligned}
$$

\subsection{The Dirichlet Prior}

Given the details from the previous section, one could easily obtain an estimate for $\hat{\rho}_{j, k}$ by setting $\pi_{a, b}$ to be proportional to $c_{a, b}$, the number of samples found in cell $(a, b)$ in the grid. 
There are two issues with this approach.

(1) This would give us a point estimate for $\hat{\rho}_{j, k}$, but no idea of the accuracy of the estimate.

(2) In reality, we know that with a small sample of the data, there are likely cells that contain data that we simply missed. We might not want to assume that the density is zero in this case. That is, though the simple estimate for each $\pi_{a, b}$ is unbiased, it does not easily incorporate prior knowledge about the distribution of data in cells.

For these two reasons, we adopt a Bayesian approach to estimating $\hat{\rho}_{j, k}$. Let $\mathbf{C}$ denote the matrix such that $c_{a, b}$ is the number of sampled points that fall in cell $(a, b)$. We view the assignment of samples to cells as taking a sample from a Multinomial distribution so that

\section{$\mathbf{C} \sim \operatorname{Multinomial}(m, \pi)$.}

Here, $m$ is the number of data points sampled for this particular $(j, k)$ pair. Furthermore, we put a user-defined $\operatorname{Dirichlet}(\beta)$ prior on $\pi$. This prior allows the user to specify hisor-her beliefs about how sparse the grid is by manipulating $\beta .^{1}$

In standard Bayesian fashion, after observing $\mathbf{C}$, one can use the conjugacy of the Dirichlet and the Multinomial [Lee 2012] to obtain the following posterior distribution on $\pi$ :

$$
\pi_{\mid \mathbf{C}} \sim \operatorname{Dirichlet}(\mathbf{C}+\beta) .
$$

This in turn provides us with a way to obtain confidence bounds on $\hat{\rho}_{j, k}$. We simply take a number of samples from a $\operatorname{Dirichlet}(\mathbf{C}+\beta)$ distribution to obtain possible values of $\pi$, and for each of them, we obtain a separate estimate for $\hat{\rho}_{j, k}$. All of these estimates give us an empirical distribution for the posterior distribution of $\hat{\rho}_{j, k}$ after seeing $\mathbf{C}$. This empirical distribution serves the same purpose as the Normal confidence bounds in the case of Fisher's method.

\subsection{Implementation}

The producer thread for the gridded approach that is responsible for actually performing the sampling can be implemented exactly as in the case of Fisher's method.

The consumer thread is quite similar to the consumer thread for Fisher's except that rather than computing the two statistics that are required for Fisher's, we use the method described above to take $m$ samples to obtain an empirical distribution for the posterior distribution of $\hat{\rho}_{j, k}$ after seeing the samples.

All of these samples are then sorted using a distributed sort, and again they are processed at a central location from highest to lowest, using a line sweeping algorithm that is very similar to the one employed when Fisher's is used. The main differences are as follows.

(1) The sweeping line now stops at each sampled correlation, since we have multiple samples from the posterior distribution of each correlation.

(2) For a particular correlation, instead of using the cumulative normal distribution, we calculate probabilities such as the probability that "the $i$ th correlation corresponding to $\Lambda^{\prime}$ is in reality $\leq c$ " by counting samples that are past $c$. In this case, the fraction of the samples that are $\leq c$ can be taken as an estimate for the desired probability.

\footnotetext{
${ }^{1}$ Note that we have (somewhat unconventionally) used matrix-valued inputs and outputs to and from the Dirichlet and Multinomial distributions. We assume that both distributions operate on matrices the same way as they do on vectors; the matrix should simply be seen as an alternative storage structure that is convenient for exposition because of the underlying grid.
} 


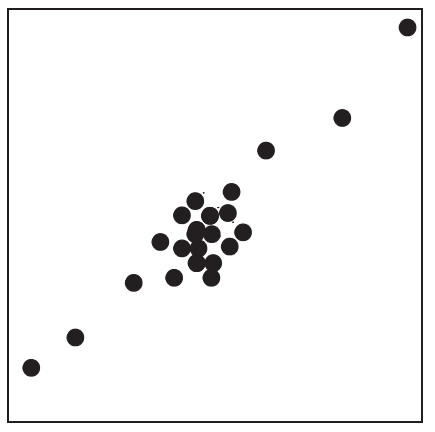

(a) Original data

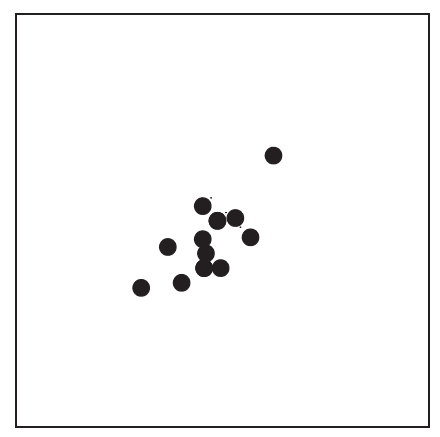

(b) Sampled data

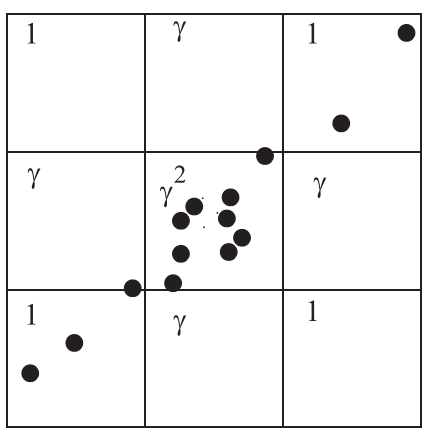

(c) Biased sampling

Fig. 5. Biased sampling. At left, we depict a set of points with a very strong correlation, and in the middle, the result of taking a sample of those points. Clearly, the apparent correlation has been weakened because we have dropped the outliers in the top and bottom corners. We can address this by breaking the data space into a set of regions, depicted on the right. In the corner regions, all points are selected (the probability of sampling a point is 1 . At the edges, it is $\gamma$, and in the middle it is $\gamma^{2}$. In this way, the points in the "middle" of the dataset have the lowest sampling fraction and expectedly, the sample is a better representation of the correlation in the data.

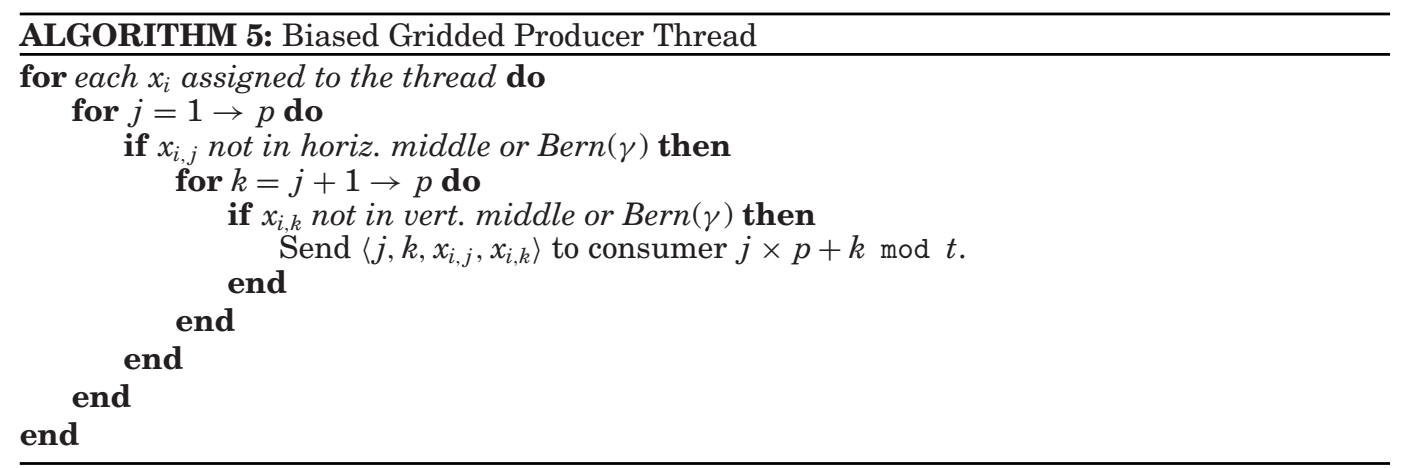

\subsection{A Biased Sampling Scheme}

One issue with the approach proposed in this section is that it is vulnerable to outliers. Points in the extreme corner of the data space can have a huge effect on the correlation-much greater than points in the middle-and yet if there are just a few of them, they are likely to be missed by any simple, uniform sampling scheme.

We can solve this by utilizing a biased sampling scheme, as illustrated in Figure 5. Before sampling, the data space is partitioned into nine regions; a parameter $\gamma \leq 1$ controls the precise sampling plan, but whatever the value for $\gamma$, points in the corners are always sampled. As $\gamma$ becomes smaller, points in the center of the data space are under-sampled to a greater and greater extent. The producer thread for this sampling scheme is implemented as follows.

Note that this bias is in addition to the gridding described in the previous few sections. Each region of the dataset will be recursively subdivided into its own grid. That is, our count matrix $\mathbf{C}$ now has nine different sub-matrices $\mathbf{C}[1], \mathbf{C}[2], \ldots, \mathbf{C}[9]$. Likewise, our density matrix $\pi$ now has nine different sub-matrices $\pi[1], \pi[2], \ldots, \pi[9]$. Finally, we need a vector $\mathbf{n}$ telling how many points in the database were in each of the nine different regions (we will discuss how to compute $\mathbf{n}$ in a moment). Rather than generating $\pi$ as a whole, as we did in Section 6.2, we now generate the portion of $\pi$ 


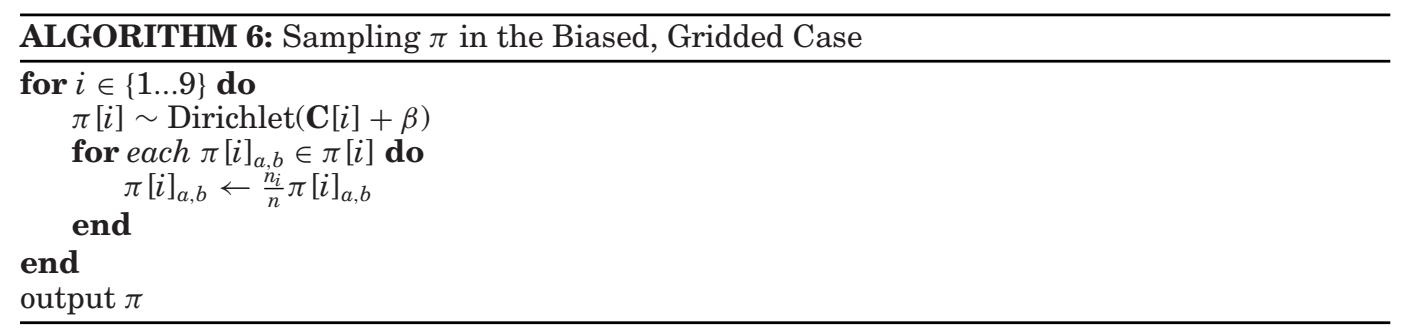

covering each of the nine regions independently, and normalize the various probabilities by taking into account the actual number of data points that fell in each region. Given this, the procedure for sampling a value from the posterior distribution for $\pi$ is as follows.

Basically, this algorithm applies the Dirichlet/Multinomial conjugacy separately to each of the nine different regions to construct $\pi$. Furthermore, it normalizes all of the $\pi[i]_{a, b}$ in region $i$ by multiplying each by the fraction of the data points that actually fell in that cell.

Once this algorithm has been used to generate a $\pi$ matrix, we can use the machinery from Section 6.1, without modification, in order to produce a sampled value for $\hat{\rho}_{j, k}$. Everything else stays the same.

Computing the number of points in each region. Algorithm 6 requires that we be able to compute $n_{1}, n_{2}, \ldots, n_{9}$. One way to do this would be to scan the entire dataset, counting the number of points in each. Unfortunately, doing this for every (dimension, dimension) pair is simply infeasible, and we need to be a bit more clever.

To compute these values efficiently, as we are scanning the data, we compute the total number of data points in each horizontal and vertical strip. Since there are only $6 p$ of those values for $p$-dimensional data, this is not a tremendous burden. This gives us

$$
\begin{gathered}
n_{1}+n_{2}+n_{3}=\Sigma_{1} \\
n_{4}+n_{5}+n_{6}=\Sigma_{2} \\
n_{7}+n_{8}+n_{9}=\Sigma_{3},
\end{gathered}
$$

for the three horizontal strips, and

$$
\begin{gathered}
n_{1}+n_{4}+n_{7}=\Sigma_{4} \\
n_{2}+n_{5}+n_{8}=\Sigma_{5} \\
n_{3}+n_{6}+n_{9}=\Sigma_{6},
\end{gathered}
$$

for the three vertical strips. In addition, because we are computing the set of points in the four corners exactly, we have

$$
n_{1}=\Sigma_{7}, n_{3}=\Sigma_{8}, n_{7}=\Sigma_{9}, n_{9}=\Sigma_{10} .
$$

In total, this gives us a very simple set of linear equations to solve, allowing us to compute each $n_{i}$ (see Figure 6).

\subsection{Choosing the Boundaries}

We close this section by considering the problem of choosing the various region and grid boundaries.

Given a set of nine regions for a particular (dimension, dimension) pair, it is easy to build a grid within each of the regions. We simply do an equi-width partitioning along 

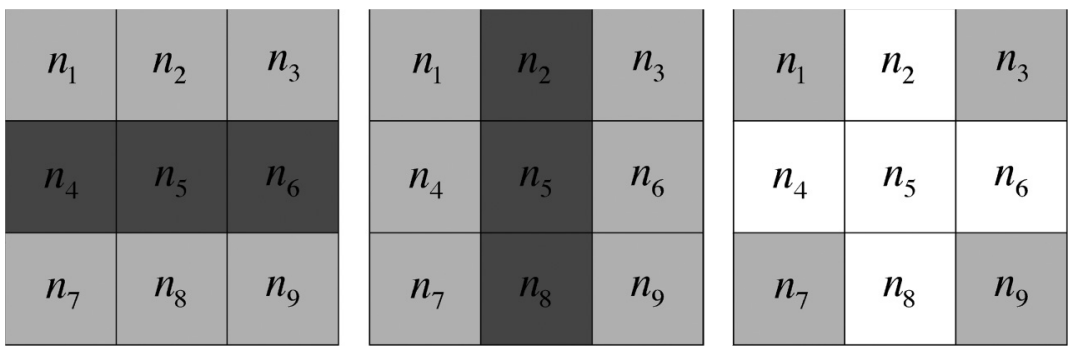

Fig. 6. The 10 different constraints that allow us to compute $n_{i}$ for each of the 9 regions.

both dimensions. In all of our experiments, the grid within a region is 50 cells wide by 50 cells tall, resulting in 2,500 cells total.

Choosing regions is more challenging. We are given the desired number of samples from a dimension pair; this value is $m$. The goal is to find the quartiles and the value of $\gamma$ that gives us the highest accuracy, subject to the constraint that the total number of samples processed for a given (dimension,dimension) pair is approximately $m$.

Recall that the dataset has $n$ points. On each dimension, we break the data into three quartiles: [0: $\left.\frac{s}{n}\right),\left[\frac{s+1}{n}: \frac{n-s}{n}\right]$, and $\left(\frac{n-s+1}{n}: 1\right] . s$ is the number of data in the top or bottom quartile, and can range from $1 \ldots \frac{\sqrt{m n}}{2}$ (the upper bound guarantees $\gamma>0$ ). [0: $\frac{s}{n}$ ) means that if there are many points that are all the sth smallest in the dataset, then they are not included in the bottom quartile; they instead are included in the middle quartile. The next smallest value is the largest one that is included in the bottom quartile. This means that the $\left[0: \frac{s}{n}\right)$ quartile can never have more than $s$ points. Likewise, $\left(\frac{n-s+1}{n}: 1\right]$ means that if there are many points that are all the $s$ th largest, they are included in the middle quartile, and in that case, the upper quartile only includes only those values that are larger than the $s$ th largest. So the $\left(\frac{n-s+1}{n}: 1\right]$ quartile can also never have more than $s$ points.

For a given dimension pair and a specific value of $s$, we can count the exact number of points in the four corner cells. Let this number be $n_{c}$. If $n_{c}$ is larger than $m$, then that value of $s$ can simply be discarded. If not, assuming that the data is independently distributed, the expected number of samples for a given dimension pair is going to be approximately $m^{\prime}=n_{c}+\frac{4 p s(n-2 s)}{n}+\frac{p^{2}(n-2 s)^{2}}{n}$. The third term is the number of points we will sample from the middle cell. And the second term is the number of points we will sample from the remaining four cells. Thus, for a given value of $s$, we can set $m=m^{\prime}$ and solve for $\gamma$ to get the required sampling proportion to obtain $m$ samples overall:

$$
\gamma=\frac{\sqrt{\left(m-n_{c}\right) n+4 s^{2}}-2 s}{n-2 s} .
$$

The crucial is to find the optimal value of $s$ for the dimension. To do this, we randomly choose 10 dimension pairs from the dataset whose correlation matrix we are interested in. For each of the 10 dimension pairs and a particular value of $s$, we simulate the sampling process from that dataset several times, compute a posterior distribution using the methods of Section 6.2, and then compute the error of the posterior distribution. Here, the error can be a squared one over the difference between a sample from the posterior distribution and the real value of the correlation. We then average the 10 errors. This average error measures the "goodness" of a particular value for $s$. Low error means high goodness. Our task is to search for the best $s$ in $1 \ldots \frac{\sqrt{m n}}{2}$. This is done 
by moving the $s$ value by a fixed step along the dimension and choosing the one that leads to the smallest average squared error.

Once we have $s$ and $\gamma$, we can apply our gridded model on each dimension pair. It is also worth mentioning that, simply increasing the number of dimension pairs when estimating the average error will not necessarily increase the top- $k$ hit rate eventually, as the data distribution for each dimension pair is different, and we just want to find a particular value for $s$ that can generally work well.

\section{EXPERIMENTAL RESULTS}

In this section, we benchmark the various algorithms that we have proposed in the article.

\subsection{Experimental Goals}

We have two main goals in benchmarking these algorithms.

(1) To see under what conditions-including dataset sizes and dataset dimensionalities - the various algorithms perform well in terms of compute time, and when they do not.

(2) To test the accuracy of the two sampling-based methods: Do they actually return a set of (dimension, dimension) pairs $\Lambda^{\prime}$ that is a close approximation of the set $\Lambda$ ? Is the accuracy dataset dependent?

\subsection{Datasets Considered}

We consider three different datasets in our experiments.

Normal. This is a $10^{4}$-dimensional dataset with $10^{6}$ data points created by sampling repeatedly from a normal distribution with $10^{4}$ dimensions. The covariance matrix used to produce the data has 10, 1,000 by 1,000 blocks of non-zero correlations along the diagonal of the matrix. The rest of the entries in the covariance matrix are zero. In theory, this should be an easy dataset because of the covariance matrix' sparsity. There are small number of strong correlations, and the rest are zero. Any two dimensions of this data are similar to Figure 3(a) in shape.

Wikipedia. This set consists of 4,135,219 Wikipedia documents. We use this dataset at three different dimensionalities: $10^{3}, 10^{4}$, and $4 \times 10^{4}$. For each dimensionality, we use the most common words in the corpus to form a feature vector. The value of the dimension associated with word $w$ is computed using a log-likelihood ratio (LLR) statistic [Dunning 1993] that measures how strongly the number of occurrences of the word in the document differs from what was expected. Assume that $n_{b}$ is the total number of word occurrences in the corpus, $m_{b}$ is the number of occurrences of $w$ in the corpus, $n_{i}$ is the total number of words in the input document, and $m_{i}$ is the number of occurrences of $w$ in the input corpus. Then, we have

$$
\operatorname{LLR}(w)=m_{i}\left(\log p_{b}-\log p_{i}\right)+\left(n_{i}-m_{i}\right)\left[\log \left(1-p_{b}\right)-\log \left(1-p_{i}\right)\right] .
$$

We stress that preforming the thresholding task for such a dataset is a very difficult task. At $4 \times 10^{4}$ dimensions, the covariance matrix has 80 million entries, and each of those entries is constructed over 4 million documents. Around $10^{15}$ floating point operations are required to compute this matrix exactly.

To give the reader an idea of the nature of this dataset, consider Figure 7, which shows the joint distribution of two arbitrarily selected dimensions. Note that the figure is in log-log scale, which is a testament to the highly skewed nature of this data. 


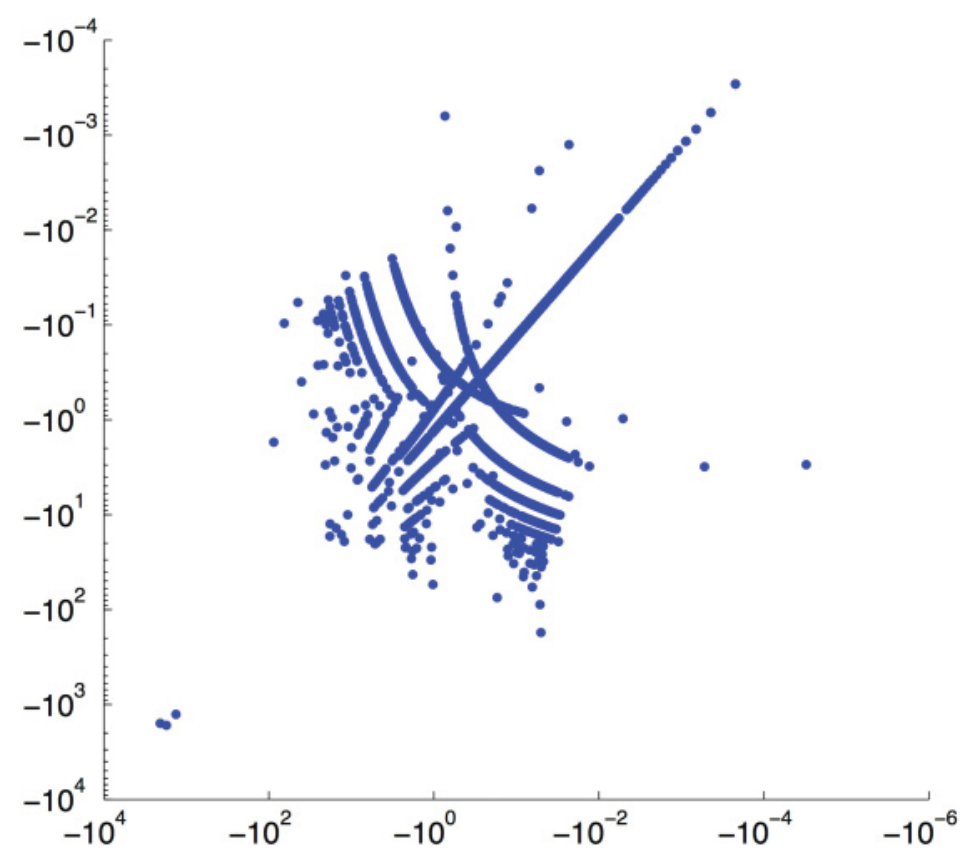

Fig. 7. Plot of two dimensions of the text dataset. Note that the plot is in log-log scale. The data are highly skewed and non-normal.

NIST. This dataset consists of 1,613,707 text documents provided by NIST. Again we use this dataset at three different dimensionalities: $10^{3}, 10^{4}$, and $4 \times 10^{4}$, and we prepare the dataset in the same way as the Wikipedia dataset was prepared.

\subsection{Hardware and Software}

All of the experiments in this article were performed using Hadoop deployed on 100 Amazon EC2 m2.4x large machines, each of which had eight virtual cores, two disks, and 68GB of RAM. All programs were written to run as series of MapReduce jobs in Java.

\subsection{Experiments Run}

We ran three experiments.

Experiment one: Performance as a function of dimensionality. For the first experiment, we tested the exact method, Fisher's, and the Gridded approach on each of the three datasets. For the two sampling-based methods, we tested both 100 and 1,000 samples per (dimension, dimension) pair. In all experiments, $K$ was set to be the dimensionality of the data. For the sampling-based methods, $\alpha$ was set to be 0.05 . The results are reported in Table I.

Experiment two: Performance as a function of dataset size. For the second experiment, we again tried all three of the methods that we proposed, again trying 100 and 1,000 samples for both Fisher's and the Gridded approaches. However, this time we used only the $10^{4}$-dimensional Wikipedia dataset. We created three additional versions of the Wikipedia dataset by adding the dataset to itself once, then twice, and, finally, thrice. This gives us the $1 \times, 2 \times, 3 \times$, and $4 \times$ versions of the Wikipedia dataset. The largest has more than 16 million documents. This experiment is meant to test scalability. The results are reported in Table II. 
Table I. The Runtime Used by Each Method Under Varying Dimensionalities

\begin{tabular}{|c|c|c|c|c|c|c|c|c|}
\hline \multirow{2}{*}{$\begin{array}{c}\text { Runtime } \\
\text { (hh:mm:ss) }\end{array}$} & Synthetic & \multicolumn{3}{c|}{ Wikipedia } & \multicolumn{3}{c|}{ NIST } \\
\cline { 2 - 9 } & $10 \mathrm{~K}$ dims & $1 \mathrm{~K}$ dims & $10 \mathrm{~K}$ dims & 40K dims & $1 \mathrm{~K}$ dims & $10 \mathrm{~K}$ dims & $40 \mathrm{~K}$ dims \\
\hline \multicolumn{2}{|c|}{ Exact } & $1: 07: 37$ & $0: 05: 10$ & $2: 53: 07$ & N/A & $0: 04: 40$ & $1: 13: 10$ & N/A \\
\hline \multirow{2}{*}{ Gridded } & 100 samples & $1: 22: 31$ & $0: 14: 18$ & $1: 49: 41$ & $19: 32: 15$ & $0: 12: 35$ & $1: 36: 51$ & $18: 54: 36$ \\
\cline { 2 - 9 } & $1 \mathrm{~K}$ samples & $1: 45: 20$ & $0: 14: 04$ & $2: 07: 47$ & $25: 00: 21$ & $0: 12: 52$ & $1: 52: 38$ & $23: 58: 01$ \\
\hline \multirow{2}{*}{ Fisher's } & 100 samples & $0: 09: 10$ & $0: 04: 14$ & $0: 09: 44$ & $0: 50: 02$ & $0: 03: 34$ & $0: 09: 17$ & $0: 43: 13$ \\
\cline { 2 - 9 } & $1 \mathrm{~K}$ samples & $0: 20: 27$ & $0: 04: 08$ & $0: 20: 16$ & $6: 10: 18$ & $0: 03: 31$ & $0: 20: 40$ & $5: 41: 58$ \\
\hline
\end{tabular}

For both datasets with $4 \times 10^{4}$ dimensions, the exact method could not be run.

Table II. The Runtime Used by Each Method As We Scale the $10^{4}$-Dimensional Wikipedia Dataset

\begin{tabular}{|c|c|c|c|c|c|}
\hline \multicolumn{2}{|c|}{$\begin{array}{c}\text { Runtime } \\
\text { (hh:mm:ss) }\end{array}$} & 1xWikipedia & 2xWikipedia & 3xWikipedia & 4xWikipedia \\
\hline \multirow{2}{*}{ Exact } & $2: 53: 07$ & $3: 58: 20$ & $5: 07: 11$ & $6: 19: 24$ \\
\hline \multirow{2}{*}{ Gridded } & 100 samples & $1: 49: 41$ & $1: 51: 43$ & $1: 52: 50$ & $1: 55: 45$ \\
\cline { 2 - 6 } & 1K samples & $2: 07: 47$ & $2: 10: 42$ & $2: 14: 54$ & $2: 17: 27$ \\
\hline \multirow{2}{*}{ Fisher's } & 100 samples & $0: 09: 44$ & $0: 09: 46$ & $0: 09: 55$ & $0: 09: 53$ \\
\cline { 2 - 6 } & 1 K samples & $0: 20: 16$ & $0: 21: 22$ & $0: 20: 58$ & $0: 21: 39$ \\
\hline
\end{tabular}

Table III. The Accuracy for Each Method, Measured by the Fraction of the Correct Top-K Covered by the Answer Set Returned ( $K$ is Equal to the Number of Dimensions in Our Experiments)

\begin{tabular}{|c|c|c|c|c|c|c|c|c|}
\hline \multirow{2}{*}{\multicolumn{2}{|c|}{$\begin{array}{c}\text { Hit-rate } \\
(\%)\end{array}$}} & Synthetic & \multicolumn{3}{|c|}{ Wikipedia } & \multicolumn{3}{|c|}{ NIST } \\
\hline & & $10 \mathrm{~K}$ dims & $1 \mathrm{~K} \operatorname{dims}$ & $10 \mathrm{~K}$ dims & $40 \mathrm{~K}$ dims & $1 \mathrm{~K} \operatorname{dims}$ & $10 \mathrm{~K}$ dims & $40 \mathrm{~K} \mathrm{dims}$ \\
\hline \multicolumn{2}{|c|}{ Exact } & 100 & 100 & 100 & \multirow{5}{*}{ N/A } & 100 & 100 & \multirow{5}{*}{ N/A } \\
\hline \multirow{2}{*}{ Gridded } & 100 samples & 78.89 & 85.5 & 28.28 & & 88.8 & 47.88 & \\
\hline & 1K samples & 99.91 & 97.1 & 95.31 & & 99.1 & 96.52 & \\
\hline \multirow{2}{*}{ Fisher's } & 100 samples & 97.64 & 9.9 & 0.54 & & 8.6 & 2 & \\
\hline & 1K samples & 100 & 24.1 & 1.06 & & 83 & 2.58 & \\
\hline
\end{tabular}

The hit rate for the exact method is always $100 \%$. Since the exact method is unable to run at $4 \times 10^{4}$ dimensions, the hit rates cannot be measured at that size.

Experiment three: Accuracy. Finally, we tested the ability of the two sampling-based methods to return correct results. We repeated the tests of Experiment One, but this time we checked how accurate the results were: what percentage of the (dimension, dimension) pairs in $\Lambda$ are actually found in the returned answer $\Lambda^{\prime}$. Unfortunately, since the exact method did not scale up to $4 \times 10^{4}$ dimensions, we were unable to obtain a ground truth at that scale. The results are reported in Table III.

\subsection{Discussion}

There are a few interesting findings that we point out now.

Scalability in dimensionality. First off, we consider the runtimes of the various methods with respect to dimensionality. All three methods scaled approximately quadratically when increasing data dimensionality from $10^{4}$ to $4 \times 10^{4}$. This is not surprising, given that all of the algorithms are quadratic with respect to $p$. Moving from $10^{3}$ to $10^{4}$ dimensions did not have nearly as dramatic effect on the running time, presumably because at that scale, the dimensionality is small enough that other costs dominate.

Fisher's was much faster than the Gridded method. This is not surprising, given that the Gridded method does a lot more work. The Gridded requires taking many samples from the appropriate posterior distribution for each of the (dimension, dimension) pairs and shuffling them; this is difficult computationally but it is especially I/O heavy, since it creates a lot of data that must be sent around the network. In contrast, Fisher's method need only send a couple of values for each such pair. Hence, it is dramatically faster at all dimensionalities. 
But perhaps the most interesting results are related to the exact method. The exact method is fast at $10^{3}$ dimensions. At $10^{4}$ dimensions the Gridded method is comparable with the exact method in terms of running time. But the exact method cannot be used at all at $4 \times 10^{4}$ dimensions. There are many reasons for this failure, as we have outlined in this article. The RAM requirements and computational requirements are simply too great for the exact method at this problem size. As we have pointed out, the time complexity of the exact method is not only proportional to $O\left(p^{2}\right)$, but also proportional to $O(n)$, which can be comparable to $O\left(p^{2}\right)$. That gives us a total of $O\left(p^{4}\right)$. On the other hand, the time complexity of the gridded method is approximately $O\left(K n+m p^{2}\right)$. As $p$ gets larger (though $K, n$, and $m$ stay stable), there is much more computation to suffer for the exact method than for the gridded method. Therefore, the exact algorithm scales much more poorly and fails to finish for $4 \times 10^{4}$ dimensions.

Scalability in dataset size. The results in Table II are as expected, but still interesting. Since the two sampling-based methods take 100 (or 1,000) samples per (dimension, dimension) pair, no matter the size of the dataset, and processing those samples are the major computational and I/O cost, they are relatively unaffected by an increase in dataset size. Hence, we have only a very tiny, linear increase in running time with increasing dataset size for the Gridded method (about 2-3 minutes per additional Wikipedia), and no discernible increase at all for Fisher's. The only additional cost to the sampling-based methods is that the implementation must scan all of the data, hence a tiny increase in running time with dataset size.

The exact method, in contrast, adds more than hour with each additional Wikipedia, as it clearly suffers from the $O\left(n p^{2}\right)$ computation.

Accuracy and sample-size selection. The Gridded method is outperformed computationally by Fisher's, but as we see in Table III, Fisher's method is very inaccurate on the real datasets. At $10^{4}$ dimensions, we found that only a few percent of the (dimension, dimension) pairs corresponding to the actual top $K$ correlations were returned by Fisher's. The results are so poor that it calls into question the utility of the method, unless the user has a very strong reason to suspect that his or her data are normally distributed. In contrast, the Gridded method returned more than $95 \%$ of the (dimension, dimension) pairs corresponding to the top $K$ correlations in every case. If the data are normally distributed (as is the case with the synthetic dataset), Fisher's is a great option, giving nearly $100 \%$ of the (dimension, dimension) pairs corresponding to the real top $K$ correlations. If not, then the Gridded method is definitely preferred.

We close this section with a discussion of sample-size selection, which is intimately connected with the issue of accuracy.

As we defined the problem addressed by the two sampling-based methods, there should only be a small chance of $\alpha=0.05$ that any of the (dimension, dimension) pairs corresponding to the top $K$ correlations will be missed, regardless of the sample size that is selected. If this were actually the case, then the only effect of changing the sample size would be a change in running time. On one hand, a larger sample size increases the running time of the correlation estimation for each (dimension, dimension) pair, but decreases the running time of the actual thresholding. The reason for the decrease in thresholding time is that larger samples shrink the confidence intervals of the estimated correlations, and so the thresholding algorithms of Section 4.5 will need to compute the exact correlations for fewer (dimension, dimension) pairs. On the other hand, a very small sample size results in a fast covariance estimation for each (dimension, dimension) pair, but will require an exact computation of the correlation for a very large number of (dimension, dimension) pairs, because the confidence bounds on each estimated correlation are so large. 
What we find in practice is that the time for the second step-the exact correlation computation-dominates the overall running time only for very, very small sample sizes (note that in our experiments, the 100-sample runs were always much faster than the 1,000-sample runs). Hence, if there is truly a $\alpha=0.05$ chance that any of the (dimension, dimension) pairs corresponding to the top $K$ correlations will be missed regardless of sample size, the optimal sample size would be very small, just big enough that the thresholding time is kept from dominating the overall computation time.

In practice, however, we found that only Fisher's method using 1,000 samples on the synthetic dataset managed to achieve $100 \%$ accuracy, despite the fact that in each case, there should only be a $\alpha=0.05$ that any of the top $K$ correlations would be missed. If things were working in practice as well as they should work in theory, we might expect to have a large number of $100 \%$ accuracy findings in Table III, which is obviously not the case.

There are a few obvious explanations for this mismatch of theory and practice. First, the Gridded method does make a number of assumptions, including the key assumption that data are uniformly distributed within a grid cell. This could cause some degradation in accuracy, as could an inappropriate choice of prior hyperparameters in conjunction with the Gridded method. In the case of Fisher's, it might be that at a very small sample size of 100 , there is simply not enough data for the asymptotic normality of the $z$ score to be reliable. As the sample size nears 1,000 , then the asymptotics kick in and the accuracy approaches $100 \%$.

What then, does all of this say about an appropriate choice of sample size in practice? We have generally found that by the time 1,000 samples have been taken, the individual (dimension, dimension) correlation estimates-and, most importantly, the confidence bounds on each individual estimate accuracy, which ultimately govern the overall accuracy of the method-become quite reliable. This is even the case in the text-based datasets whose test results we report in this article, and these datasets seem to be particularly nasty due to the extreme skew (see Figure 7). For less than 1,000 samples, the individual confidence bounds can be inaccurate. Thus, 1,000 samples is a reasonable rule of thumb.

If one desires a more adaptive sampling rate that reflects the actual characteristics of a particular dataset, the number of samples could be chosen on a case-by-case basis by first randomly selecting a small number of (dimension, dimension) pairs (say, 1,000 of them) and computing an exact correlation for each selected pair, by scanning the entire dataset. Then, one could try various sampling rates and choose the smallest sampling rate for which sampling-based confidence bounds are found to be accuratethat is, for a particular sampling rate, one could compute $95 \%$ confidence bounds on each correlation, and find the smallest rate for which approximately 950 out of 1,000 true correlations are within the bounds. This would result in a high-quality choice for the number of samples to take.

\section{CONCLUSIONS}

In this article, we considered the problem of regularizing a large covariance matrix efficiently in a distributed fashion over a large dataset. We began by considering thresholding through the exact algorithm in detail, pointing out where it will and will not be feasible. We then considered reducing the time complexity using sampling-based methods to compute the thresholded covariance matrix. Our algorithms have probabilistic bounds which imply that with high probability, all of the top $K$ entries in the matrix have been retained. We described a set of experiments on a 100-machine cluster that shed some light on the relative merits of the proposed methods. 


\section{REFERENCES}

Peter J. Bickel and Elizaveta Levina. 2008a. Covariance regularization by thresholding. The Annals of Statistics 36, 6 (2008), 2577-2604.

Peter J. Bickel and Elizaveta Levina. 2008b. Regularized estimation of large covariance matrices. The Annals of Statistics 36, 1 (2008), 199-227.

Tony Cai and Weidong Liu. 2011. Adaptive thresholding for sparse covariance matrix estimation. Journal of the American Statistical Association 106, 494 (2011), 672-684.

Tony Cai, Weidong Liu, and Xi Luo. 2011. A constrained 1 minimization approach to sparse precision matrix estimation. Journal of the American Statistical Association 106, 494 (2011), 594-607.

Surajit Chaudhuri, Rajeev Motwani, and Vivek Narasayya. 1998. Random sampling for histogram construction: How much is enough? In Proceedings of ACM SIGMOD Record, Vol. 27. ACM, 436-447.

Edith Cohen, Mayur Datar, Shinji Fujiwara, Aristides Gionis, Piotr Indyk, Rajeev Motwani, Jeffrey D. Ullman, and Cheng Yang. 2001. Finding interesting associations without support pruning. IEEE Transactions on Knowledge and Data Engineering 13, 1 (2001), 64-78.

Jeffrey Dean and Sanjay Ghemawat. 2008. MapReduce: Simplified data processing on large clusters. Communications of the ACM 51, 1 (2008), 107-113.

P. H. Diananda. 1953. Some probability limit theorems with statistical applications. In Proceedings of the Cambridge Philosophical Society, Vol. 49. Cambridge University Press, 239-246.

Ted Dunning. 1993. Accurate methods for the statistics of surprise and coincidence. Computational Linguistics 19, 1 (1993), 61-74.

Jianqing Fan, Yingying Fan, and Jinchi Lv. 2008. High dimensional covariance matrix estimation using a factor model. Journal of Econometrics 147, 1 (2008), 186-197.

Jianqing Fan, Yuan Liao, and Martina Mincheva. 2011. High dimensional covariance matrix estimation in approximate factor models. Annals of Statistics 39, 6 (2011), 3320.

Ronald A. Fisher and others. 1915. Frequency distribution of the values of the correlation coefficient in samples from an indefinitely large population. Biometrika 10, 4 (1915), 507-521.

Jerome H. Friedman. 1989. Regularized discriminant analysis. Journal of the American Statistical Association 84, 405 (1989), 165-175.

Reinhard Furrer and Thomas Bengtsson. 2007. Estimation of high-dimensional prior and posterior covariance matrices in Kalman filter variants. Journal of Multivariate Analysis 98, 2 (2007), 227-255.

Gösta Grahne, Laks V. S. Lakshmanan, and Xiaohong Wang. 2000. Efficient mining of constrained correlated sets. In Proceedings of the International Conference on Data Engineering (ICDE 2000). IEEE, $512-521$.

William Gropp, Ewing Lusk, and Anthony Skjellum. 1999. Using MPI: Portable Parallel Programming with the Message-Passing Interface, Vol. 1. MIT Press.

Dominique Guillot and Bala Rajaratnam. 2012. Retaining positive definiteness in thresholded matrices. Linear Algebra and its Applications 436, 11 (2012), 4143-4160.

Peter J. Haas and Arun N. Swami. 1992. Sequential sampling procedures for query size estimation. In Proceedings of the ACM SIGMOD Record, Vol. 21, 341-350.

Cho-Jui Hsieh, Mátyás A. Sustik, Inderjit Dhillon, Pradeep Ravikumar, and Russell Poldrack. 2013. BIG \& QUIC: Sparse inverse covariance estimation for a million variables. In Proceedings of the Advances in Neural Information Processing Systems (NIPS). 3165-3173.

Cho-Jui Hsieh, Matyas A. Sustik, Inderjit S. Dhillon, and Pradeep D. Ravikumar. 2011. Sparse inverse covariance matrix estimation using quadratic approximation. In Proceedings of the Advances in Neural Information Processing Systems (NIPS). 2330-2338.

Jianhua Z. Huang, Naiping Liu, Mohsen Pourahmadi, and Linxu Liu. 2006. Covariance matrix selection and estimation via penalised normal likelihood. Biometrika 93, 1 (2006), 85-98.

Ihab F. Ilyas, Volker Markl, Peter Haas, Paul Brown, and Ashraf Aboulnaga. 2004. CORDS: Automatic discovery of correlations and soft functional dependencies. In Proceedings of SIGMOD 2004. ACM, $647-658$.

Alan Julian Izenman. 2008. Linear Discriminant Analysis. Springer.

Binyan Jiang. 2013. Covariance selection by thresholding the sample correlation matrix. Statistics \& Probability Letters 83, 11 (2013), 2492-2498.

Iain M. Johnstone. 2001. On the distribution of the largest eigenvalue in principal components analysis. Annals of Statistics 29, 2 (2001), 295-327.

Ian Jolliffe. 2005. Principal Component Analysis. Wiley Online Library. 
Ross Kindermann, James Laurie Snell, and others. 1980. Markov Random Fields and Their Applications, Vol. 1. American Mathematical Society Providence, RI.

Mladen Kolar and Eric P. Xing. 2012. Consistent covariance selection from data with missing values. In Proceedings of ICML.

Daphne Koller and Nir Friedman. 2009. Probabilistic Graphical Models: Principles and Techniques. MIT Press.

Clifford Lam and Jianqing Fan. 2009. Sparsistency and rates of convergence in large covariance matrix estimation. Annals of Statistics 37, 6B (2009), 4254.

Peter M. Lee. 2012. Bayesian Statistics: An Introduction. John Wiley \& Sons.

Elizaveta Levina, Adam Rothman, Ji Zhu, and others. 2008. Sparse estimation of large covariance matrices via a nested Lasso penalty. The Annals of Applied Statistics 2, 1 (2008), 245-263.

Richard J. Lipton, Jeffrey F. Naughton, and Donovan A. Schneider. 1990. Practical selectivity estimation through adaptive sampling. In Proceedings of the 1990 ACM SIGMOD International Conference on Management of Data, Vol. 19. ACM.

Vladimir A. Marčenko and Leonid Andreevich Pastur. 1967. Distribution of eigenvalues for some sets of random matrices. Sbornik: Mathematics 1, 4 (1967), 457-483.

Peder A. Olsen, Figen Öztoprak, Jorge Nocedal, and Steven J. Rennie. 2012. Newton-like methods for sparse inverse covariance estimation. In Proceedings of the Advances in Neural Information Processing Systems (NIPS). 764-772.

Niketan Pansare, V. R. Borkar, Chris Jermaine, and Tyson Condie. 2011. Online aggregation for large mapreduce jobs. In Proceedings of the VLDB Endowment, Vol. 4. 1135-1145.

Benjamin T. Rolfs, Bala Rajaratnam, Dominique Guillot, Ian Wong, and Arian Maleki. 2012. Iterative thresholding algorithm for sparse inverse covariance estimation. In Proceedings of the Advances in Neural Information Processing Systems (NIPS). 1583-1591.

Adam J. Rothman, Elizaveta Levina, and Ji Zhu. 2009. Generalized thresholding of large covariance matrices. Journal of the American Statistical Association 104, 485 (2009), 177-186.

David I. Warton. 2008. Penalized normal likelihood and ridge regularization of correlation and covariance matrices. Journal of the American Statistical Association 103, 481 (2008), 340-349.

Christopher Williams and Matthias Seeger. 2001. Using the Nyström method to speed up kernel machines. In Proceedings of the 14th Annual Conference on Neural Information Processing Systems. 682-688.

Hui Xiong, Mark Brodie, and Sheng Ma. 2006. Top-cop: Mining top-k strongly correlated pairs in large databases. In Proceedings of International Conference on Data Mining (ICDM'06). IEEE, 1162-1166.

Lingzhou Xue, Shiqian Ma, and Hui Zou. 2012. Positive-definite 1-penalized estimation of large covariance matrices. Journal of the American Statistical Association 107, 500 (2012), 1480-1491.

Ming Yuan and Yi Lin. 2007. Model selection and estimation in the Gaussian graphical model. Biometrika 94, 1 (2007), 19-35.

Received May 2014; revised May 2015; accepted May 2016 